\title{
ON THE NORMAL BUNDLES OF RATIONAL CURVES ON FANO 3-FOLDS*
}

\author{
MINGMIN SHEN ${ }^{\dagger}$
}

\begin{abstract}
A component of very free rational curves on a variety is called unbalanced if the normal bundle of a general member is unbalanced. In this paper we show that all components of very free rational curves on a Fano threefold of Picard number one are balanced with the only exception being the space of conics on $\mathbb{P}^{3}$.
\end{abstract}

Key words. Rational curves, Fano threefolds.

AMS subject classifications. Primary 14M22; Secondary 14D15.

1. Introduction. In this paper, we work over the field $\mathbb{C}$ of complex numbers. A variety $X$ is Fano if it is smooth projective with the anticanonical class, $-K_{X}$, being ample. It is known that Fano varieties are rationally connected and hence contain a lot of rational curves, see [4] and [24]. The geometry of the space of rational curves carries a lot of information of the variety itself. Unfortunately, some basic questions concerning moduli space of rational curves on a Fano variety are still open. For example, let $X$ be a smooth Fano variety of Picard number one. Let $M_{e}=M_{e}(X)$ be the space of degree $e$ rational curves on $X$. One can ask the following

QUESTION 1.1. Is $M_{e}$ irreducible, at least for $e$ sufficiently large?

We see that the answer is "yes" for $X=\mathbb{P}^{n}$. A positive answer for $X$ being a quadric hypersurface follows from [22] or [35]. The case of cubic threefolds is treated in [33] and cubic hypersurfaces of higher dimensions are treated in [6]. In [15], the authors give a positive answer to the above question for a general hypersurface $X \subset \mathbb{P}_{\mathbb{C}}^{n}$ with degree $d<\frac{n-1}{2}$.

In this paper, we consider a variation of the above question. Let $X$ be a smooth projective variety. Assume that $\operatorname{dim} X \geq 3$. Let $M \subset \overline{\mathcal{M}}_{0,0}(X, \beta)$ be a component of the Kontsevich moduli space of genus 0 curves on $\mathrm{X}$. Assume that for a general member $[C] \in M$, the corresponding rational curve $\phi: \mathbb{P}^{1} \cong C \rightarrow X$ is birational onto image and very free. Recall that $\phi$ being very free means that $\phi^{*} T_{X}$ is ample, see [23]. We call $M$ a component of very free rational curves on $X$. Then it follows that $\phi: C \rightarrow X$ is a closed immersion for general $[C] \in M$. The normal bundle of a general such curve $C$ in $X$ has splitting type

$$
\mathscr{N}_{C / X} \cong \mathcal{O}\left(a_{1}\right) \oplus \mathcal{O}\left(a_{2}\right) \oplus \cdots \oplus \mathcal{O}\left(a_{n-1}\right), \quad n=\operatorname{dim} X,
$$

with $1 \leq a_{1} \leq a_{2} \leq \cdots \leq a_{n-1}$. The sequence $\left(a_{1}, \ldots, a_{n-1}\right)$ is an invariant of the component $M$.

Definition 1.2. We say that $M$ (and also $\mathscr{N}_{C / X}$ ) is balanced if $a_{n-1}-a_{1} \leq 1$ and that $M$ (and also $\mathscr{N}_{C / X}$ ) is unbalanced if $a_{n-1}-a_{1} \geq 2$.

Question 1.3. For a Fano variety $X$ of Picard number one, is $M_{e}$ balanced (for sufficiently large $e$ )?

\footnotetext{
*Received May 24, 2011; accepted for publication June 3, 2011.

†DPMMS, University of Cambridge, Wilberforce Road, Cambridge, CB3 0WB, UK (M.Shen@ dpmms.cam.ac.uk).
} 
The deformation of a curve on $X$ is controlled by its normal bundle and Question 1.3 is essentially asking for the normal bundle of a general rational curve on $X$. If the normal bundle is balanced, then its splitting type is completely determined by its degree. Without the restriction on Picard number, there could be a lot of unbalanced rational curves, for example when $X=\mathbb{P}^{1} \times \mathbb{P}^{n}$. But when $X$ has Picard number one, we do not expect a general rational curve to move much more freely in some direction than in others. In this paper, we carry out this idea in the three dimensional case.

Assume that a smooth projective threefold $X / \mathbb{C}$ has an unbalanced component $M$ of very free rational curves. Then a general member of $M$ has unbalanced normal bundle, so it moves more freely in some direction. By deforming the curve in this direction, we get a surface $\Sigma$ (the construction is given in section 2 in a more general setting). Actually we also show that the unbalancedness gives a canonical foliation on $M$ which is algebraically integrable with a leaf being all curves lying on a fixed $\Sigma$. In section 3 , we study those surfaces $\Sigma$. There are two different types of surface $\Sigma$ that we can get. Accordingly, $M$ is either of conic type or fibration type, see Definition 3.16. If $M$ is of conic type, then $C \subset X$ is étale locally equivalent to a conic in $\mathbb{P}^{3}$; If $M$ is of fibration type and $-K_{X}$ is nef, then there is a rational component $S$ of rational curves on $X$ with trivial normal bundle, see Theorem 3.15. After that, we focus on the case when $X$ is Fano of Picard number one. In section 5 , we show that the Abel-Jacobi mapping defined by $S$ is never trivial as long as $X$ has nonzero intermediate jacobian; this shows that $S$ can not be rational. The main theorem of this paper is the following

TheOrem 1.4. Let $X$ be a Fano threefold of Picard number one. If $X$ has an unbalanced component $M$ of very free rational curves, then $X=\mathbb{P}^{3}$ and $M$ is the space of conics on $X$.

The intermediate jacobian of $X$ is zero only if $X=X_{5}$ or $X=X_{22}$. The cases $X=X_{5}$ and $X=X_{22}$ are ruled out by a ramification argument. The case $X_{5}$ follows from the paper [11]. The author carries out the construction of the space of conics on $X_{22}$ in the appendix. After this was done, Prof. J. Kollár informed the author of the paper [25], where a similar construction is carried out using a different model of $X_{22}$. We note that the above theorem gives a new characterization of $\mathbb{P}^{3}$. Namely, $\mathbb{P}^{3}$ would be the only Fano threefold of Picard number one which has an unbalanced component of very free rational curves.

Acknowledgement. This work is the main part of my $\mathrm{PhD}$ thesis. I would like to thank my advisor, Aise Johan de Jong, for his careful and patient instructions. Several discussions with Jason Starr and Matt DeLand were also very helpful and Professor Friedman assured me of some results about intermediate jacobians. I would like to thank them all for their help.

2. Basic constructions. Notations and assumptions: Let $X$ be a smooth projective variety over the field $\mathbb{C}$ of complex numbers. Assume that $\operatorname{dim} X=n$. Let $M \subset \overline{\mathcal{M}}_{0,0}(X, \beta)$ be an unbalanced component of very free rational curves on $X$. For a general member $[C] \in M$, we assume that the splitting type of the normal bundle to be

$$
\mathscr{N}_{C / X} \cong \mathcal{O}(a)^{\oplus(n-r-1)} \oplus \mathcal{O}\left(b_{1}\right) \oplus \cdots \oplus \mathcal{O}\left(b_{r}\right)
$$


where $3 \leq a+2 \leq b_{1} \leq \cdots \leq b_{r}$. Let $M^{0} \subset M$ be the open subscheme parametrizing smooth rational curves $C \cong \mathbb{P}^{1} \subset X$ with normal bundle (1). Let

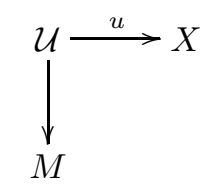

be the universal family over $M$. For any $[C] \in M$, let $u_{[C]}: C \rightarrow X$ denote the corresponding morphism. We say that $C$ passes through a point $P \in X$ or that $P$ is on $C$ if $P$ is on the image of $u_{[C]}$. Let $P_{i} \in X, i=1,2, \ldots, k$, be distinct points on $X$. We define

$$
M^{0}\left(P_{1}, \ldots, P_{k}\right) \subset M^{0}
$$

to be the subscheme that consists of $[C] \in M^{0}$ such that $C$ passes through all the points $P_{i}$. We use $\mathcal{U}^{0}\left(P_{1}, \ldots, P_{k}\right) \rightarrow X$ to denote the universal family over $M^{0}\left(P_{1}, \ldots, P_{k}\right)$. Let $[C] \in M^{0}\left(P_{1}, \ldots, P_{k}\right)$. The obstruction of deforming $C$ in $X$ passing through $\left\{P_{1}, \ldots, P_{k}\right\}$ is in $\mathrm{H}^{1}\left(C, \mathscr{N}_{C / X}\left(-P_{1}-\cdots-P_{k}\right)\right)$. Since $\mathscr{N}_{C / X}$ has splitting type (1) for $[C] \in M^{0}$, we know that $M^{0}\left(P_{1}, \ldots, P_{k}\right)$ is smooth when $k \leq a+1$.

DEFinition 2.1. Notations and assumptions as above, we use

$$
\operatorname{Def}_{X}\left(C ; P_{1}, \ldots, P_{k}\right) \subset M^{0}\left(P_{1}, \ldots, P_{k}\right)
$$

to denote the union of the irreducible components of $M^{0}\left(P_{1}, \ldots, P_{k}\right)$ containing $[C]$. And we get the corresponding universal family.

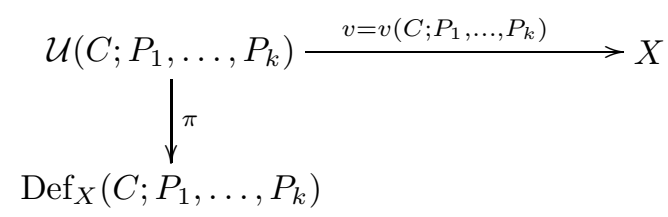

Let

$$
\sigma_{i}: \operatorname{Def}_{X}\left(C ; P_{1}, \ldots, P_{k}\right) \rightarrow \mathcal{U}\left(C ; P_{1}, \ldots, P_{k}\right)
$$

be the section that gets contracted by $v$ to the point $P_{i} \in X$, where $i=1, \ldots, k$. We use the notation $\Sigma\left(C ; P_{1}, \ldots, P_{k}\right) \subset X$ to denote the closure of the image of $v\left(C ; P_{1}, \ldots, P_{k}\right)$.

REMARK 2.2. If $[C]$ is a smooth point of $M^{0}\left(P_{1}, \ldots, P_{k}\right)$, for example when $k \leq a+1$, then $\operatorname{Def}_{X}\left(C ; P_{1}, \ldots, P_{k}\right)$ is irreducible. Actually, it is smooth everywhere if $k \leq a+1$.

In our situation, let $[C] \in M^{0}$ and we take $k=a+1$ and pick $\left\{P_{1}, \ldots, P_{a+1}\right\}$ to be distinct points on $C$, hence $\operatorname{Def}_{X}\left(C ; P_{1}, \ldots, P_{a+1}\right)$ is smooth. Note that the Zariski tangent space $T_{p}$ of $\mathcal{U}\left(C ; P_{1}, \ldots, P_{k}\right)$ at $p \in \pi^{-1}\left(\left[C^{\prime}\right]\right)$ fits into the following short exact sequence naturally

$$
0 \longrightarrow T_{C^{\prime}, p} \longrightarrow T_{p} \stackrel{d \pi}{\longrightarrow} \mathrm{H}^{0}\left(C^{\prime}, \mathscr{N}^{\prime}(-a-1)\right) \longrightarrow 0
$$


for any point $\left[C^{\prime}\right] \in \operatorname{Def}_{X}\left(C ; P_{1}, \ldots, P_{a+1}\right)$, where $\mathscr{N}^{\prime}=\mathscr{N}_{C^{\prime} / X}$. Consider the differential of $v$ at $p$, we have the following

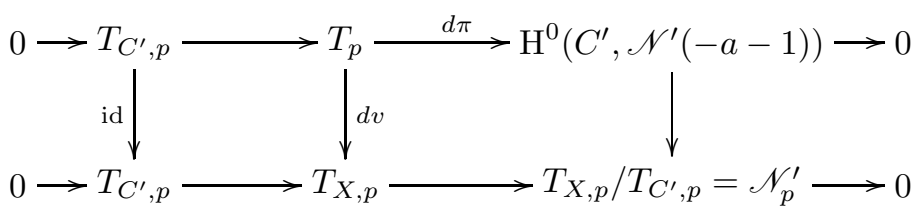

Here the last column is the evaluation of a section at the point $p$. It is easy to see that $d v$ has rank $r+1$ if $p \notin \sigma_{i}$ for all $i=1, \ldots, a+1$. Let $\Sigma=\Sigma\left(C ; P_{1}, \ldots, P_{a+1}\right) \subset X$ and we have $\operatorname{dim} \Sigma=r+1$ since everything is over $\mathbb{C}$. Let $\phi: \Sigma^{\prime} \rightarrow \Sigma$ be the normalization of $\Sigma$ and $\tilde{\phi}: \tilde{\Sigma} \rightarrow \Sigma^{\prime}$ be a resolution of $\Sigma^{\prime}$. To make further constructions, we need the following

LEMMA 2.3. Let $f: U \rightarrow V$ be a morphism between smooth algebraic varieties over an algebraically closed field $k$ of characteristic 0 . Assume that $d f$ is generically of rank $r$. Let $\Sigma \subset V$ be the closure of $f(U)$ and $\Sigma^{\prime}$ be the normalization of $\Sigma$. Then $f$ naturally lifts to $f^{\prime}: U \rightarrow \Sigma^{\prime}$

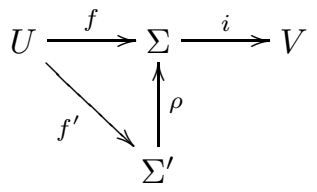

and for any closed point $x \in U$ with $d f(x)$ having rank $r, \Sigma^{\prime}$ is smooth at $f^{\prime}(x)$ and $d f^{\prime}$ has rank $r$ at $x$.

Proof. The existence of $f^{\prime}$ is easy since $U$ is smooth and hence normal. We only need to prove the remaining part. Let $y=f(x) \in \Sigma \subset V$ and $y^{\prime}=f^{\prime}(x)$. The problem is local, so we can choose an $r$ dimensional closed subvariety $Z \subset U$ which is smooth at $x$ and $d f(x)$ is injective on $T_{Z, x} \otimes k(x)$. We can replace $U$ by $Z$. So we assume that $U$ has dimension $r$. We have the following local homomorphisms between local rings.

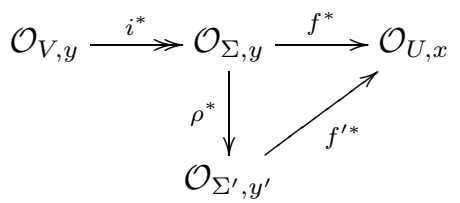

Choose a set of local parameters $\left\{t_{1}, \ldots, t_{n}\right\}$ of $V$ at $y$ such that $\left\{t_{1}, \ldots, t_{r}\right\}$ pull back to local parameters of $U$ at $x$. So we get the following diagram

$$
k\left[t_{1}, \ldots, t_{r}\right] \hookrightarrow \mathcal{O}_{\Sigma^{\prime}, y^{\prime}} \hookrightarrow \mathcal{O}_{U, x}
$$

with $\mathcal{O}_{\Sigma^{\prime}, y^{\prime}}$ being an intermediate normal domain of an étale ring extension. After taking the completions, we get a splitting

$$
k\left[\left[t_{1}, \ldots, t_{r}\right]\right] \hookrightarrow \hat{\mathcal{O}}_{\Sigma^{\prime}, y^{\prime}} \rightarrow k\left[\left[t_{1}, \ldots, t_{r}\right]\right]
$$

with the composition being identity. So $\hat{\mathcal{O}}_{\Sigma^{\prime}, y^{\prime}}=k\left[\left[t_{1}, \ldots, t_{r}\right]\right] \oplus I$, where $I$ is an ideal of $\hat{\mathcal{O}}_{\Sigma^{\prime}, y^{\prime}}$ and a module over $k\left[\left[t_{1}, \ldots, t_{r}\right]\right]$. By the analytical irreducibility and 
analytical normality of normal varieties, (see [38]), we know that $\hat{\mathcal{O}}_{\Sigma^{\prime}, y^{\prime}}$ is an integrally closed integral domain of dimension $r$. This forces $I$ to be zero. Indeed for any $a \in I$, by dimension reason, $a$ should be algebraic over $k\left[\left[t_{1}, \ldots, t_{r}\right]\right]$. Let

$$
f_{n} a^{n}+f_{n-1} a^{n-1} \cdots+f_{0}=0, \quad \text { with } \quad f_{i} \in k\left[\left[t_{1}, \ldots, t_{r}\right]\right]
$$

be an equation for $a$ with minimal degree. Then $f_{0}$ is also in $I$ and hence has to be 0 . This means $a=0$.

Apply the above lemma to $v: \mathcal{U}^{\prime} \rightarrow X$ and we get

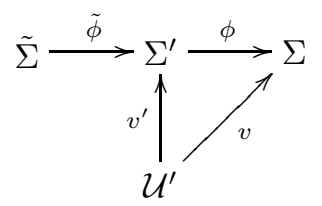

where $\mathcal{U}^{\prime}=\mathcal{U}\left(C ; P_{1}, \ldots, P_{a+1}\right)$. Let $\mathcal{U}_{0}^{\prime}:=\mathcal{U}^{\prime}-\cup_{i=1}^{a+1} \sigma_{i}$, where $\sigma_{i}$ is the section that gets contracted to $P_{i}$. Then we also know that the image $v^{\prime}\left(\mathcal{U}_{0}^{\prime}\right)$ is in the smooth locus of $\Sigma^{\prime}$ and $\left.v^{\prime}\right|_{\mathcal{U}_{0}^{\prime}}$ is a smooth morphism. Pick an arbitrary point $\left[C^{\prime}\right] \in \operatorname{Def}_{X}\left(C ; P_{1}, \ldots, P_{k}\right)$. If we restrict the above maps to $\left[C^{\prime}\right]$, we get

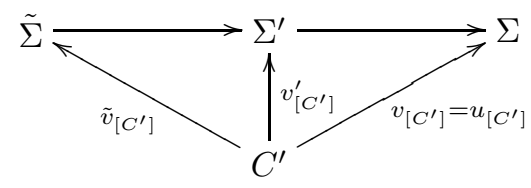

Note that $\operatorname{Def}_{X}\left(C ; P_{1}, \ldots, P_{a+1}\right)$ remains the same if we replace $C$ by $C^{\prime}$. From now on, by abuse of notation, we will use $C$ in stead of $C^{\prime}$ to denote an arbitrary curve from the family $\mathcal{U}\left(C ; P_{1}, \ldots, P_{a+1}\right)$.

Lemma 2.4. For all points $Q \in C-\left\{P_{1}, \ldots, P_{a+1}\right\}, \Sigma^{\prime}$ is smooth at $v_{[C]}^{\prime}(Q)$. The morphism $\tilde{\Sigma} \rightarrow \Sigma^{\prime}$ is an isomorphism along $C-\left\{P_{1}, \ldots, P_{a+1}\right\}$.

Proof. Indeed, we already see that $v^{\prime}\left(\mathcal{U}_{0}^{\prime}\right)$ is in the smooth locus of $\Sigma^{\prime}$.

Proposition 2.5. For any $[C] \in M^{0}$, the variety $\Sigma=\Sigma\left(C ; P_{1}, \ldots, P_{a+1}\right)$ is independent of the choice of $\left\{P_{1}, \ldots, P_{a+1}\right\}$.

Proof. Consider the morphism $v^{\prime}: \mathcal{U}^{\prime}=\mathcal{U}\left(C ; P_{1}, \ldots, P_{a+1}\right) \rightarrow \Sigma^{\prime}$. Let $Q$ be a point of $C$ that is different from the $P_{i}$ 's. Since $\Sigma^{\prime}$ is smooth at $Q^{\prime}=v_{[C]}^{\prime}(Q)$ and $v^{\prime}$ is smooth above the point $Q^{\prime}$, we get $Z=v^{\prime-1}\left(Q^{\prime}\right)$ is smooth. Let $Z_{0}$ be the component of $Z$ that contains the point $Q$ above $[C]$. Note that $\mathcal{U}^{\prime}$ is a smooth irreducible component of $\mathcal{U}^{0}\left(P_{1}, \ldots, P_{a+1}\right)$. Hence $\pi\left(Z_{0}\right)$ becomes a component of $M^{0}\left(P_{1}, \ldots, P_{a+1}, Q\right)$ on which $[C]$ is a smooth point. By definition we have $Z_{0} \cong$ $\pi\left(Z_{0}\right) \cong \operatorname{Def}_{X}\left(C ; P_{1}, \ldots, P_{a+1}, Q\right)$. Consider the universal family

$$
w: \mathcal{U}^{\prime \prime}=\mathcal{U}\left(C ; P_{1}, \ldots, P_{a+1}, Q\right) \rightarrow X
$$

Since $\mathcal{U}^{\prime \prime} \subset \mathcal{U}^{\prime}$ and $w=\left.v\right|_{\mathcal{U}^{\prime \prime}}$, we see that the image of $w$ is contained in $\Sigma$. Hence we get $w: \mathcal{U}^{\prime \prime} \rightarrow \Sigma$. Note that $Z_{0}$ is smooth and hence $\mathcal{U}^{\prime \prime}$ is smooth. So we can lift $w$ 
to get $w^{\prime}: \mathcal{U}^{\prime \prime} \rightarrow \Sigma^{\prime}$. By dimension count, we have

$$
\begin{aligned}
\operatorname{dim} Z_{0} & =\operatorname{dim} \mathcal{U}^{\prime}-\operatorname{dim} \Sigma^{\prime} \\
& =\operatorname{dim} \mathrm{H}^{0}\left(C, \mathscr{N}_{C / X}(-a-1)\right)+1-(r+1) \\
& =\Sigma_{i=1}^{r}\left(b_{i}-a\right)-r \\
& =\operatorname{dim} \mathrm{H}^{0}\left(C, \mathscr{N}_{C / X}(-a-2)\right)
\end{aligned}
$$

This means that the deformation of $C$ in $X$ with the points $\left\{P_{1}, \ldots, P_{a+1}, Q\right\}$ fixed is actually unobstructed. Then we can use a same argument to show that the rank of $d w^{\prime}$ is generically $r$ as we did for $d v^{\prime}$. This implies $\Sigma=\Sigma\left(C ; P_{1}, \ldots, P_{a+1}, Q\right)$. Then we have

$$
\Sigma\left(C ; P_{1}, \ldots, P_{a+1}\right)=\Sigma\left(C ; P_{1}, \ldots, P_{a}, P_{a+1}, P_{a+1}^{\prime}\right)=\Sigma\left(C ; P_{1}, \ldots, P_{a}, P_{a+1}^{\prime}\right)
$$

and hence by induction we can replace $\left\{P_{i}\right\}_{i=1}^{a+1}$ by another set of $a+1$ points. Thus $\Sigma$ is independent of the choice of $\left\{P_{i}\right\}_{i=1}^{a+1}$.

Definition 2.6. Let $\varphi: U \rightarrow V$ be a morphism between smooth varieties, we define the normal sheaf of the morphism to be

$$
\mathscr{N}_{U / V}=\mathscr{N}_{\varphi}=\operatorname{coker}\left(d \varphi: T_{U} \rightarrow \varphi^{*} T_{V}\right) .
$$

We say that $\varphi$ has injective tangent map at a closed point $x \in U$ if $d \varphi(x)$ is injective. Note that $\mathscr{N}_{\varphi}$ is locally free at points where $\varphi$ has injective tangent map.

COROllary 2.7. Assume that $[C] \in M^{0}$. Then the variety $\Sigma^{\prime}$ is smooth along $v_{[C]}^{\prime}(C) \cong C$ and $\mathscr{N}_{C / \Sigma^{\prime}} \cong \mathcal{O}\left(b_{1}\right) \oplus \cdots \oplus \mathcal{O}\left(b_{r}\right)$. The normal bundle $\mathscr{N}_{\tilde{\Sigma} / X}$ is locally free along the curve $\tilde{v}_{[C]}(C) \cong C$ and $\left.\mathscr{N}_{\tilde{\Sigma} / X}\right|_{C} \cong \mathcal{O}(a)^{\oplus(n-r-1)}$.

Proof. Let $Q \in C$ be an arbitrary point. Pick a set of $a+1$ distinct points $\left\{P_{1}, \ldots, P_{a+1}\right\}$ that are different from $Q$. Then by Proposition 2.5, we have $\Sigma=\Sigma\left(C ; P_{1}, \ldots, P_{a+1}\right)$. By Lemma 2.4 , we know that $\Sigma^{\prime}$ is smooth at $v_{[C]}^{\prime}(Q)$. Consider the deformation of $v_{[C]}: C \rightarrow \Sigma^{\prime}$ with the points $\left\{P_{1}, \ldots, P_{a+1}\right\}$ fixed. Such deformations still form a covering family. This means that $\mathscr{N}_{C / \Sigma^{\prime}}(-a-1)$ is globally generated. Assume that

$$
\mathscr{N}_{C / \Sigma^{\prime}} \cong \mathcal{O}\left(b_{1}^{\prime}\right) \oplus \cdots \oplus \mathcal{O}\left(b_{r}^{\prime}\right)
$$

then we get $b_{i}^{\prime} \geq a+1$ for all $i=1, \ldots, r$. We first show that $\mathscr{N}_{\tilde{\Sigma} / X}$ is locally free along $C$. For any point $x \in C$, pick a set $\left\{P_{1}, \ldots, P_{a+1}\right\}$ of $a+1$ distinct points on $C$ that are different from the given point $x$. Then we get the morphism

$$
v: \mathcal{U}^{\prime}=\mathcal{U}\left(C ; P_{1}, \ldots, P_{a+1}\right) \rightarrow X
$$

which factors through $v^{\prime}: \mathcal{U}^{\prime} \rightarrow \Sigma^{\prime}$. Correspondingly we have the induced maps between Zariski tangent spaces at $x$,

$$
T_{\mathcal{U}^{\prime}, x} \stackrel{d v^{\prime}(x)}{\longrightarrow} T_{\Sigma^{\prime}, v^{\prime}(x)} \stackrel{d \phi\left(v^{\prime}(x)\right)}{\longrightarrow} T_{X, v(x)}
$$

and the composition is exactly $d v(x)$. We already know that $d v(x)$ and $d v^{\prime}(x)$ have rank $r+1$. Together with the fact that $\operatorname{dim} T_{\Sigma^{\prime}, x}=r+1$, we know that $d \phi\left(v^{\prime}(x)\right)$ is 
injective. So $\phi$ has injective tangent map along $C$. Note that $\tilde{\Sigma} \rightarrow \Sigma^{\prime}$ is isomorphism along $C$. Hence $\mathscr{N}_{\tilde{\Sigma} / X}$ is locally free of rank $n-r-1$ along $C$. Now we consider the following short exact sequence

$$
\left.0 \rightarrow \mathscr{N}_{C / \tilde{\Sigma}} \stackrel{\eta}{\longrightarrow} \mathscr{N}_{C / X} \stackrel{\theta}{\longrightarrow} \mathscr{N}_{\tilde{\Sigma} / X}\right|_{C} \longrightarrow 0
$$

Since $b_{i}^{\prime} \geq a+1$, the image of $\eta$ lies in the summand $\sum_{i=1}^{r} \mathcal{O}\left(b_{i}\right)$. Then we get the following diagram

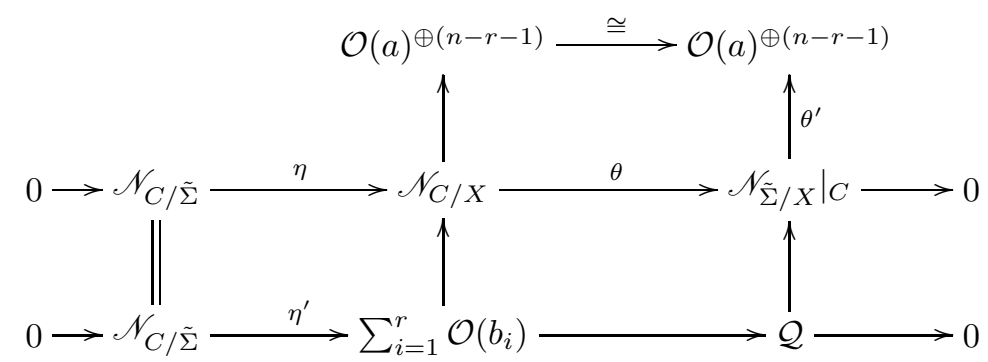

where the second and third columns are also short exact sequences and $\mathcal{Q}$ is a torsion sheaf. We have already shown that $\left.\mathscr{N}_{\tilde{\Sigma} / X}\right|_{C}$ is locally free of rank $n-r-1$. This forces $\mathcal{Q}$ to be 0 . Hence $\eta^{\prime}$ and $\theta^{\prime}$ are isomorphisms.

Definition 2.8. Given $[C] \in M^{0}$, let $\Sigma, \Sigma^{\prime}$ and $\tilde{\Sigma}$ be as above. Since the morphism $v_{[C]}: C \rightarrow X$ lifts to $\tilde{v}_{[C]}: C \rightarrow \tilde{\Sigma}$, we can define $\beta^{\prime}$ to be the homology class of $C$ on $\tilde{\Sigma}$. Let $\operatorname{Def}_{\tilde{\Sigma}} \subset \overline{\mathcal{M}}_{0,0}\left(\tilde{\Sigma}, \beta^{\prime}\right)$ be the space of curves $C \cong \mathbb{P}^{1} \rightarrow \tilde{\Sigma}$ such that the composition $C \rightarrow \tilde{\Sigma} \rightarrow X$ is a point on $M^{0}$. Hence we can view $\tilde{v}_{[C]}$ as a point on $\operatorname{Def}_{\tilde{\Sigma}}$. By abuse of notation, we still use $[C]$ to denote this point. Let $\operatorname{Def}_{\tilde{\Sigma}}(C) \subset \operatorname{Def}_{\tilde{\Sigma}}$ be the irreducible component that contains the point $[C]$. From the corollary above, we know that $\operatorname{Def}_{\tilde{\Sigma}}(C)$ is actually a smooth open subscheme of $\overline{\mathcal{M}}_{0,0}\left(\tilde{\Sigma}, \beta^{\prime}\right)$. By composing $C \rightarrow \tilde{\Sigma}$ with $\tilde{\Sigma} \rightarrow X$, we have a morphism between smooth varieties $\alpha: \operatorname{Def}_{\tilde{\Sigma}}(C) \rightarrow M^{0}$.

Proposition 2.9. The morphism $\alpha$ is a closed immersion. Furthermore, there is a nonempty open subscheme $U \subset M^{0}$ and a smooth morphism $\psi: U \rightarrow B$ such that for any $[C] \in U$ we have $\psi^{-1}(\psi([C]))=D e f_{\tilde{\Sigma}}(C) \cap U$. The quotient $B$ is smooth of dimension $(a+1)(n-r-1)$.

Proof. For simplicity, we set $D(C)=\operatorname{Def}_{\tilde{\Sigma}}(C)$. First, we show that $\alpha$ separates points. If $C_{1}$ and $C_{2}$ on $\tilde{\Sigma}$ map to the same $C$ on $X$, then $\Sigma$ has two branches along the curve $C$. But this is impossible since in the definition of $\Sigma$, the nearby deformation of $C$ should swipe out a unique branch of $\Sigma$. Now we prove that the differential $d \alpha(t)=d \alpha \otimes k(t)$ is injective for all closed points $t \in D(C)$ and that $D(C)$ is closed in $M^{0}$. Consider the universal family $\pi^{0}: \mathcal{U}^{0} \rightarrow M^{0}$. Let $\mathscr{N}$ be the cokernel of $T_{\mathcal{U}^{0} / M^{0}} \rightarrow\left(u^{0}\right)^{*} T_{X}$ where $u^{0}: \mathcal{U}^{0} \rightarrow X$ is the universal morphism. Then by the definition of $M^{0}$, the sheaf $\mathscr{N}$ is locally free and splits uniformly along the closed fibers of $\pi^{0}$. Let $\mathscr{V}_{\eta} \subset \mathscr{N}_{\eta}$ be the part of Harder-Narasimhan filtration on the generic fiber that corresponds to $\oplus_{i=1}^{r} \mathcal{O}\left(b_{i}\right)$ on the geometric generic fiber, c.f. [16]. Since the splitting of $\mathscr{N}$ is uniform, $\mathscr{V}_{\eta}$ extends to a subbundle $\mathscr{V}$ of $\mathscr{N}$. Actually, we can write down $\mathscr{V}$ explicitly as in [3]. Let $\mathscr{D}=\left(\pi^{0}\right)_{*} \mathscr{V} \subset T_{M^{0}}=\left(\pi^{0}\right)_{*} \mathscr{N}$ be the corresponding subbundle. If we write down the the differential $d \alpha([C])$ explicitly, we have

$$
d \alpha([C]): T_{D(C),[C]}=\mathrm{H}^{0}\left(C, \mathscr{N}_{C / \tilde{\Sigma}}\right) \rightarrow T_{M^{0},[C]}=\mathrm{H}^{0}\left(C, \mathscr{N}_{C / X}\right) .
$$


Since we already see that $\mathscr{N}_{C / \tilde{\Sigma}}$ maps isomorphically onto $\left.\mathscr{V}\right|_{C}$ inside $\mathscr{N}_{C / X}=\left.\mathscr{N}\right|_{C}$. This implies that $T_{D(C),[C]}=\mathscr{D} \otimes k([C])$ and in particular, the differential $d \alpha(t)$ is injective for all closed point $t \in D(C)$. Thus $\mathscr{D}$ defines a foliation on $M^{0}$ and $D(C)=\operatorname{Def}_{\tilde{\Sigma}}(C)$ defines a leaf of $\mathscr{D}$, c.f. [9]. Let $\bar{D}(C)$ be the Zariski closure of $D(C)$ in $M^{0}$. Since $\mathscr{D}$ is a subbundle of $T_{M^{0}}$, we conclude that $\bar{D}(C)$ is smooth and still a leaf, c.f. [9] (Lemma 2.3 there). Now we claim that $D(C)=\bar{D}(C)$. Otherwise, let $\left[C^{\prime}\right] \in \bar{D}(C)$ be a point that is not contained in $D(C)$. Then both $D\left(C^{\prime}\right)$ and $\bar{D}(C)$ are leaves through $\left[C^{\prime}\right]$; they have to agree on an open part. Thus $D\left(C^{\prime}\right)$ and $D(C)$ meet each other. This can happen only when $D(C)=D\left(C^{\prime}\right)$. This means that $\left[C^{\prime}\right] \in D(C)$, which is a contradiction. Hence we proved that $\alpha$ is a closed immersion. Since all the leaves of the foliation $\mathscr{D}$ are algebraic, hence $\mathscr{D}$ is algebraically integrable. This means that there is a nonempty open $U \subset M^{0}$ and a morphism $\psi: U \rightarrow B$ such that $T_{U / B}=\left.\mathscr{D}\right|_{U}$, c.f. [9] (Proposition 2.1 there). The smoothness results are from direct local computations.

3. Three dimensional case. Situation 3.1. In this whole section, we fix the following assumptions and notations:

- $X / \mathbb{C}$ is a smooth projective algebraic variety with $\operatorname{dim} X=3$.

- $M \subset \overline{\mathcal{M}}_{0,0}(X, \beta)$ is an unbalanced component of very free rational curves on $X$. Let $M^{0} \subset M$ be as in the previous section. We always use $C$ to denote a curve on $X$ such that $[C] \in M^{0}$.

- $\mathscr{N}_{C / X} \cong \mathcal{O}(a) \oplus \mathcal{O}(b)$ with $1 \leq a \leq b-2$.

- Let $\Sigma_{C}=\Sigma\left(C ; P_{1}, \ldots, P_{a+1}\right)$ be the surface as is constructed in the previous section; Let $\Sigma_{C}^{\prime}$ be the normalization of $\Sigma_{C}$ and $\tilde{\Sigma}_{C}$ be a resolution of $\Sigma_{C}^{\prime}$. We frequently drop the subscript $C$ when there is no confusion.

Definition 3.2. ([14]) Let $C_{i} \subset X_{i}$ be a curve on a variety $X_{i}, i=1,2$. We say that $C_{1} \subset X_{1}$ is equivalent to $C_{2} \subset X_{2}$ and write $\left(C_{1} \subset X_{1}\right) \cong\left(C_{2} \subset X_{2}\right)$ if there is an open neighborhood $V_{i}$ of $C_{i}$ in $X_{i}$ and an isomorphism $f: V_{1} \rightarrow V_{2}$ with $\left.f\right|_{C_{1}}: C_{1} \rightarrow C_{2}$ being also an isomorphism.

Proposition 3.3. The pair $C \subset \Sigma^{\prime}=\Sigma_{C}^{\prime}$ is equivalent to one of the following

(i) $\sigma \subset F_{n}$, where $F_{n}=\mathbb{P}(\mathcal{O}(-n) \oplus \mathcal{O}) \rightarrow \mathbb{P}^{1}$ is the Hirzebruch surface and $\sigma$ is a section;

(ii) a smooth conic on $\mathbb{P}^{2}$.

Proof. Since we only care about a neighborhood of $C \subset \Sigma^{\prime}$, we may replace $\Sigma^{\prime}$ by $\tilde{\Sigma}$, see Lemma 2.4. Consider the complete linear system $|C|$. Since $C$ is a very free rational curve on $\tilde{\Sigma}$, we know that $\tilde{\Sigma}$ is a smooth rational surface and hence $h^{i}\left(\tilde{\Sigma}, \mathcal{O}_{\tilde{\Sigma}}\right)=0$ for $i=1,2$. From the long exact sequence associated to the following short exact sequence

$$
0 \longrightarrow \mathcal{O}_{\tilde{\Sigma}} \longrightarrow \mathcal{O}_{\tilde{\Sigma}}(C) \longrightarrow \mathcal{O}_{\mathbb{P}^{1}}(b) \longrightarrow 0
$$

we get $\operatorname{dim}|C|=b+1$. Since $h^{1}\left(\tilde{\Sigma}, \mathcal{O}_{\tilde{\Sigma}}\right)=0$, any nearby deformation of $C$ in $\tilde{\Sigma}$ is in $|C|$. Then the fact that $C$ being very free implies that $|C|$ separates points and tangent vectors along $C$. Hence $|C|$ defines an immersion $\phi=\phi_{|C|}$ on a neighborhood of $C$. Let $\bar{\Sigma} \subset \mathbb{P}^{b+1}$ be the closure of the image of $\phi$. Then $\operatorname{deg} \bar{\Sigma}=C^{2}=b$, this means that $\bar{\Sigma}$ is a surface of minimal degree. The proposition is a direct application of a theorem of Del Pezzo and Bertini (c.f.[8]). 


\subsection{Case I: Smooth conic on $\mathbb{P}^{2}$.}

SituATION 3.4. In this subsection, we make the following further assumptions in addition to Situation 3.1.

- $C \subset \Sigma_{C}^{\prime}$ is equivalent to a smooth conic on $\mathbb{P}^{2}$.

- Let $U^{\prime}=U_{C}^{\prime} \subset \Sigma^{\prime}=\Sigma_{C}^{\prime}$ be the largest open neighborhood of $C$ such that $C \subset U^{\prime}$ is isomorphic to an open neighborhood of a smooth conic on $\mathbb{P}^{2}$ and $\mathscr{N}_{U^{\prime} / X}$ is locally free.

- A curve on $U^{\prime}$ is called a line/conic if it is so when we identify $U^{\prime}$ with an open subset of $\mathbb{P}^{2}$.

LEMMA 3.5. With the above assumptions, we have $a=2$ and $b=4$.

Proof. Since $C \subset \Sigma^{\prime}$ is equivalent to a smooth conic on $\mathbb{P}^{2}$, by Lemma 2.7 , we have $\mathcal{O}(b) \cong \mathscr{N}_{C / \Sigma^{\prime}}=\mathcal{O}(4)$. Hence we have $b=4$. Then $a$ is equal to either 1 or 2 . Let $L^{\prime} \subset U^{\prime}$ be a line and assume that $\left.\mathscr{N}_{U^{\prime} / X}\right|_{L} \cong \mathcal{O}(c)$. Then $\left.\mathcal{O}(a) \cong \mathscr{N}_{U^{\prime} / X}\right|_{C} \cong \mathcal{O}(2 c)$. This implies that $a=2 c=2$ is the only possibility.

Definition 3.6. Let $L \cong \mathbb{P}^{1} \subset X$ be a smooth rational curve. We say that $L$ is a pseudo-line on $X$ if there exists some $[C] \in M^{0}$ such that $L$ is the image of a line $L^{\prime} \subset U_{C}^{\prime} \subset \mathbb{P}^{2}$. Let

$$
F(X)=\left\{[L] \in \operatorname{Hilb}(X) \mid L \cong \mathbb{P}^{1} \subset X \text { is a pseudo-line }\right\} \subset \operatorname{Hilb}(X)
$$

be the moduli space of pseudo-lines on $X$. Given point $x \in X$, let

$$
F_{x}(X)=\{[L] \in F(X) \mid x \in L\} \subset F(X)
$$

be the space of pseudo-lines on $X$ that pass through the point $x$. We use $P(X)$ and $P_{x}(X)$ to denote the universal family of pseudo-lines over $F(X)$ and $F_{x}(X)$ respectively.

REMARK 3.7. We will see from the next proposition that $F(X)$ is actually an irreducible smooth open subscheme of $\operatorname{Hilb}(X)$.

Proposition 3.8. Let $L$ be a pseudo-line on $X$. Then the following are true

(i) The normal bundle $\mathscr{N}_{L / X} \cong \mathcal{O}(1) \oplus \mathcal{O}(1)$;

(ii) The definition of a pseudo-line is independent of the choice of $L^{\prime} \subset \Sigma^{\prime}$ in the following sense: If there is another $\left[C_{1}\right] \in M^{0}$ and $L_{1}^{\prime} \cong \mathbb{P}^{1} \subset U_{C_{1}}^{\prime}$ is a rational curve whose image is a curve $L_{1} \cong \mathbb{P}^{1} \subset X$ with $\mathscr{N}_{L_{1} / X} \cong \mathcal{O}(1) \oplus \mathcal{O}(1)$, then $L_{1}^{\prime}$ is a line and hence $L_{1}$ is a pseudo-line;

(iii) Any nearby deformation of $L$ in $X$ is still a pseudo-line on $X$. Namely, if $Y \rightarrow T$ is a family of rational curves on $X$ and $Y_{t_{0}}$ is a pseudo-line, then there is a nonempty open $T^{0} \subset T$ such that $Y_{t}$ is a pseudo-line for all $t \in T^{0}$;

(iv) The space $F(X)$ is smooth and irreducible;

(v) Let $L_{1}$ and $L_{2}$ be two intersecting pseudo-lines on $X$, then they are lying on a unique $\Sigma$;

(vi) The space $F_{x}(X)$ is smooth and irreducible;

(vii) Through a general pair of points on $X$, there are only finitely many pseudolines. 
Proof. (i) Let $L^{\prime} \subset U^{\prime}$ be the line that maps to $L$. Since $\left.\mathscr{N}_{U^{\prime} / X}\right|_{C} \cong \mathcal{O}(a)=\mathcal{O}(2)$, we get $\left.\mathscr{N}_{U^{\prime} / X}\right|_{L^{\prime}} \cong \mathcal{O}(1)$. Since $\mathscr{N}_{L^{\prime} / U^{\prime}}=\mathcal{O}(1)$, part (i) of the proposition follows from the following short exact sequence

$$
\left.0 \longrightarrow \mathscr{N}_{L^{\prime} / U^{\prime}} \longrightarrow \mathscr{N}_{L / X} \longrightarrow \mathscr{N}_{U^{\prime} / X}\right|_{L^{\prime}} \longrightarrow 0
$$

(ii) Let $L_{1}^{\prime} \subset U_{C_{1}}^{\prime} \subset \Sigma_{C_{1}}^{\prime}$ be a rational curve that maps to $L_{1}$. Then we still have the corresponding short exact sequence as above. Since the left term $\mathscr{N}_{L_{1}^{\prime} / U_{C_{1}}^{\prime}}$ is ample (note that $L_{1}^{\prime}$ can be viewed as a curve on $\mathbb{P}^{2}$ ) and the middle term is still $\mathcal{O}(1) \oplus \mathcal{O}(1)$, we get $\mathscr{N}_{L_{1}^{\prime} / U_{C_{1}}^{\prime}} \cong \mathcal{O}(1)$. Hence $L_{1}^{\prime}$ is a line.

To prove (iii), let $Y \rightarrow T$ be a family of smooth curves on $X$ such that $Y_{t_{0}}$ is a pseudo-line, where $T$ is a smooth curve. We want to show that $Y_{t}$ is a pseudo-line on $X$ for general $t \in T$. We may assume that the normal bundle of $Y_{t}$ in $X$ is isomorphic to $\mathcal{O}(1) \oplus \mathcal{O}(1)$. After shrinking $T$ and replacing $T$ by a finite cover if necessary, we can find a family, $Z \rightarrow T$, of pseudo-lines on $X$ such that $Z_{t}$ and $Y_{t}$ meet at a single point and both $Z_{t_{0}}$ and $Y_{t_{0}}$ lie on the same $\Sigma^{\prime}$ associated to some $[C] \in M^{0}$. Then $Y_{t_{0}} \vee Z_{t_{0}}$ is a degeneration of $C$ and hence $\left[Y_{t_{0}} \vee Z_{t_{0}}\right] \in M$. Deformation theory tells us that the obstruction of deforming $Y_{t} \vee Z_{t}$ is in the second hyper-extension group of the cotangent complex, of the morphism $\phi: C_{t}^{\prime}=Y_{t} \vee Z_{t} \rightarrow X$, by the structure sheaf of $C_{t}^{\prime}$. Namely, the obstruction is in $\operatorname{Ext}_{\mathcal{O}_{C_{t}^{\prime}}}^{2}\left(L_{\phi}^{*}, \mathcal{O}_{C_{t}^{\prime}}\right)$, where $L_{\phi}^{*}$ is the cotangent complex of $\phi$, see [26]. A long exact sequence associated to the spectral sequence is

$$
\cdots \longrightarrow \operatorname{Ext}_{\mathcal{O}_{C_{t}^{\prime}}}^{1}\left(\phi^{*} \Omega_{X / k}^{1}, \mathcal{O}_{C_{t}^{\prime}}\right) \longrightarrow \operatorname{Ext}_{\mathcal{O}_{C_{t}^{\prime}}}^{2}\left(L_{\phi}^{*}, \mathcal{O}_{C_{t}^{\prime}}\right) \longrightarrow \operatorname{Ext}_{\mathcal{O}_{C_{t}^{\prime}}}^{2}\left(\Omega_{C_{t}^{\prime} / k}, \mathcal{O}_{C_{t}^{\prime}}\right)
$$

This shows that $Y_{t} \vee Z_{t}$ is unobstructed in $X$, see [32]. Hence $\left\{Y_{t} \vee Z_{t}: t \in T\right\}$ corresponds to a curve inside the smooth locus of $M$. To show that $Y_{t}$ is a pseudo-line, we pick points $P_{1}, P_{2} \in Y_{t}$ and $Q \in Z_{t}$ which are different from the node. The deformation of $Y_{t} \vee Z_{t}$ in $X$ passing through $P_{1}, P_{2}$ and $Q$ is still unobstructed and a general deformation gives a smooth rational curve $C_{1} \subset X$. This can be seen from the same long exact sequence as above with all the sheaves twisted by $\mathcal{O}_{C_{t}^{\prime}}\left(-P_{1}-P_{2}-Q\right)$. Hence $Y_{t}$ is on the surface $\Sigma_{1}$ that is associated to $C_{1}$. Since $Y_{t}$ is a component of the degeneration of $C_{1}$ on $\Sigma_{1}$, we get that $Y_{t}$ is a pseudo-line on $X$.

(iv) Now we know that $F(X)$ is an open subscheme of the Hilbert scheme of $X$. The smoothness follows directly from the unobstructedness of pseudo-lines on $X$. To show that $F(X)$ is irreducible, one only needs to show that it is connected. According to Proposition 2.9, there is an open subscheme $U \subset M^{0}$ with a quotient map $\psi: U \rightarrow B$. Let $\left.\mathcal{U}^{0}\right|_{U} \rightarrow X$ be the universal family over $U$. Consider the following diagram.

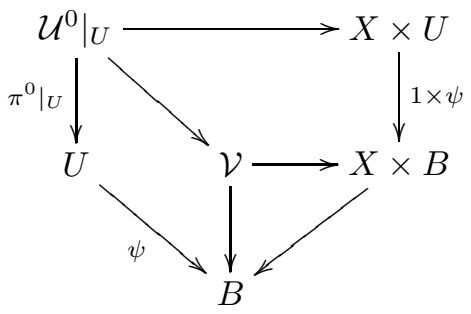

Here $\mathcal{V}$ is the closure of the image of $\left.\mathcal{U}^{0}\right|_{U}$ in $X \times B$. Let $\mathcal{V}^{\prime} \rightarrow \mathcal{V}$ be the normalization and $\tilde{\mathcal{V}} \rightarrow \mathcal{V}$ be a resolution of singularity. For each $b \in B$, there is a canonically 
associated $\Sigma_{b}$. Then for general $b \in B, \tilde{\mathcal{V}}_{b}=\tilde{\Sigma}_{b}$ and $\mathcal{V}_{b}^{\prime}=\Sigma_{b}^{\prime}$. Let $L_{1} \subset \Sigma_{b_{1}}$ and $L_{2} \subset \Sigma_{b_{2}}$ be two general pseudo-lines on $X$. By deforming $L_{1}$ in $\tilde{\mathcal{V}}$, we can connect $L_{1}$ with a pseudo-line $L_{3} \subset \Sigma_{b_{2}}$ by a one dimensional family of pseudo-lines. Note that here we use the fact that $B$ is irreducible which is a consequence of the irreducibility of $M$. By deforming $L_{2}$ inside $\Sigma_{b_{2}}^{\prime}$, we can connect $L_{2}$ with $L_{3}$ by another one dimensional family of pseudo-lines. This shows that $F(X)$ is connected.

To prove (v), let $L_{1}$ and $L_{2}$ be two intersecting pseudo-lines with intersection point $x \in X$. We only need to show that $\left[L_{1} \vee L_{2}\right] \in M$. By deforming $L_{1} \vee L_{2}$, we may assume that $x$ is a general point. Fix another pair of general intersecting pseudo-lines $\left[L_{3} \vee L_{4}\right] \in M$ with intersection point $y$. The next step is to construct a one dimensional family of pairs of intersecting pseudo-lines with $L_{1} \vee L_{2}$ and $L_{3} \vee L_{4}$ being two special fibers. Consider the universal family of pseudo-lines on $X$.

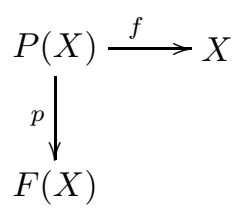

By Bertini theorem, we can find a smooth irreducible curve $\Gamma \subset X$ that passes through $x$ and $y$ such that $f^{-1}(\Gamma)$ is smooth irreducible. Note that the morphism $f^{-1}(\Gamma) \rightarrow \Gamma$ is smooth. Let $\Gamma^{\prime}$ be the normalization of $\Gamma$ inside $f^{-1}(\Gamma)$, then $f^{-1}(\Gamma) \rightarrow \Gamma^{\prime}$ has connected fibers. The pseudo-lines $L_{i}$ determine points $Q_{i} \in f^{-1}(\Gamma)$. After taking some finite covering $\tilde{\Gamma}$ of $\Gamma^{\prime}$ we may assume that there are sections $\sigma_{1}, \sigma_{2}$ of $\mathscr{C}=$ $f^{-1}(\Gamma) \times_{\Gamma^{\prime}} \tilde{\Gamma} \rightarrow \tilde{\Gamma}$ such that $Q_{1}, Q_{3} \in \sigma_{1}(\tilde{\Gamma})$ and $Q_{2}, Q_{4} \in \sigma_{2}(\tilde{\Gamma})$. By composing with the morphism $p$ in (4), each of the $\sigma_{1}$ and $\sigma_{2}$ defines a family of pseudo-lines. The two families of pseudo-lines defined by $\sigma_{i}$ give a family of intersecting pseudo-lines $Y \rightarrow \tilde{\Gamma}$ such that $L_{1} \vee L_{2}$ and $L_{3} \vee L_{4}$ are two fibers. Since $\left[L_{3} \vee L_{4}\right] \in M$, we get $\left[L_{1} \vee L_{2}\right] \in M$. The existence and uniqueness of $\Sigma$ containing $L_{1}$ and $L_{2}$ follows from deforming $L_{1} \vee L_{2}$ with three points fixed as before.

(vi) By deformation theory, we see that $F_{x}(X)$ is smooth, so we only need to show that it is connected. Let $L_{1}$ and $L_{2}$ be two pseudo-lines passing through $x$, by (v) we get a unique $\Sigma$. By deformation inside $\Sigma$, we see that there is a curve connecting $\left[L_{1}\right]$ and $\left[L_{2}\right]$ in $F_{x}(X)$.

(vii) Given two general points on $X$, all pseudo-lines passing through the two points form a zero dimensional subvariety of $F(X)$ and hence finite.

Lemma 3.9. Assume that there is a unique pseudo-line through a general pair of points on $X$, then $C \subset X$ is equivalent to a conic in $\mathbb{P}^{3}$ for $[C] \in M^{0}$ and pseudo-lines on $X$ correspond to lines on $\mathbb{P}^{3}$.

Proof. Let $U_{C} \subset \Sigma_{C}$ be the image of $U_{C}^{\prime} \subset \Sigma_{C}^{\prime}$. First we claim that under the assumption of the lemma, the surface $U_{C}$ is smooth. In fact, if $U$ is not smooth then there are two points $P_{1}, P_{2} \in U_{C}^{\prime} \subset \Sigma_{C}^{\prime}$ that map to the same point $P \in U$. Pick a general point $Q^{\prime} \in U^{\prime}$ which maps to $Q \in U$. The two lines connecting $Q^{\prime}$ with $P_{1}, P_{2}$ will give two pseudo-lines on $X$ connecting $P$ and $Q$, which is a contradiction. Then we claim that the complete linear system $|\Sigma|$ is three dimensional. Since $X$ is rationally connected, $h^{i}\left(X, \mathcal{O}_{X}\right)=0$ for $i \geq 1$. Hence rational equivalence is the same as algebraic equivalence for divisors on $X$. Let $Z \rightarrow T$ be a flat family of divisors on $X$ over a one dimensional smooth base $T$. Assume that $Z_{t_{0}}=\Sigma_{0}$ and let $C_{0}$ be a curve that defines $\Sigma_{0}$. Consider the deformation of $C_{0}$ in $Z$. By the first claim we 
know that $C_{0}$ is in the smooth locus of $Z$. Hence $C_{0}$ moves to nearby divisors. Hence $Z_{t}=\Sigma_{t}$ is swept out by some $C_{t}$ for general $t \in T$. This shows that $B \subset|\Sigma| \cong \mathbb{P}^{3}$ is an open subset, where $B$ is the quotient as in Proposition 2.9 which is three dimensional. Let $\varphi=\varphi_{|\Sigma|}: X \rightarrow \mathbb{P}^{3}$ be the map defined by the linear system $|\Sigma|$. Next we show that $\varphi$ defines an isomorphism on a neighborhood of $C$ in $X$ and maps $C$ to a smooth conic. But this is clear. Since $C$ is very free on $X$, one sees that $|\Sigma|$ separates points and also separates tangent vectors in a neighborhood of $C$. The splitting of $\mathscr{N}_{C / X}$ shows that $C$ maps to a conic on $\mathbb{P}^{3}$.

Now we return to the general case. Consider the universal family of pseudo-lines passing through a general point $x \in X$. We write $P_{x}:=P_{x}(X)$ and $F_{x}:=F_{x}(X)$. Then we have the following diagram<smiles>[Y][PH2+][Y7]</smiles>

where $\pi_{x}$ has a section $s_{x}: F_{x} \rightarrow P_{x}$, which is contracted by $f_{x}$ to the point $x$. Let $P_{x}^{0}=P_{x}-s_{x}\left(F_{x}\right)$, then $f_{x}$ is étale on $P_{x}^{0}$. Let $Y$ be the normalization of $X$ inside the function field of $P_{x}$ via $f_{x}$. Then we have the following diagram.

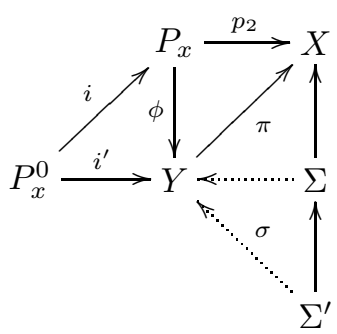

In the above diagram $i$ and $i^{\prime}$ are open immersions; $\Sigma$ is one of the surfaces that pass through $x$ and $\Sigma^{\prime}$ its normalization. The existence of $\sigma$ is due to the fact that for a general point $y \in \Sigma^{\prime}$, there is a unique line $L \subset \Sigma^{\prime}$ connecting $x$ and $y$. Since $\sigma$ is defined on an open set whose complement has at least codimension 2, we may assume that $\sigma$ is defined on a neighborhood of $C \subset \Sigma^{\prime}$ and by choosing $\Sigma$ general, we may also assume that $\sigma(C)$ is in the smooth locus of $Y$.

Proposition 3.10. Under the assumptions of Situation 3.4, there exists a normal variety $Y$ and a finite morphism $\pi: Y \rightarrow X$ with the following properties

(i) There is an open subset $V \subset X$ such that $\left.\pi\right|_{\pi^{-1}(V)}: \pi^{-1}(V) \rightarrow V$ is étale and $C \subset V$ for general $[C] \in M$. There is an open immersion $\rho: \pi^{-1}(V) \rightarrow \mathbb{P}^{3}$ such that for general $[C] \in M$ with $C \subset V$, any lifting $C \subset \pi^{-1}(V)$ is equivalent to a conic on $\mathbb{P}^{3}$ under $\rho$.

(ii) A general line $L^{\prime} \subset \pi^{-1}(V)$ maps to a pseudo-line $L \subset X$.

(iii) The degree $d=\operatorname{deg}(\pi)$ of the morphism $\pi$ is equal to the number of pseudo-lines connecting a general pair of points on $X$. The inverse image $\pi^{-1}(L)$ of a general pseudo-line $L \subset X$ is a disjoint union of d lines in $\pi^{-1}(V)$.

Proof. Pick a general point $x \in X$ and let $Y$ be the normalization of $X$ in the function field of $P_{x}$ as in (5). The proof of the proposition will be divided into several steps. 
Step 1. For general $[C] \in M^{0}$, the curve $C \subset X$ can always be lifted to a curve on $Y$ such that $\pi$ is étale along the lifting.

Proof of step 1. Pick a surface $\Sigma^{\prime}=\Sigma_{C}^{\prime}$ as in (5) with $x \in \Sigma_{C}$. Then $\sigma$ gives a lifting of $C$ to $Y$. Fix such a curve, we show that $\pi$ is étale along $\sigma(C)$. Pick an arbitrary point $x^{\prime} \in C$. We can always find a conic $C_{1}$ on $U_{C}^{\prime} \subset \Sigma_{C}^{\prime}$ such that $x, x^{\prime} \in C_{1}$ and $\left[C_{1}\right] \in M^{0}$. Hence we also get that $\Sigma_{1}:=\Sigma_{C_{1}}$ is the same as $\Sigma$ and $\sigma_{1}: \Sigma_{1}^{\prime} \rightarrow Y$ is the same as $\sigma$. It is easy to see that the image of $d \pi\left(\sigma\left(x^{\prime}\right)\right)$ : $T_{Y} \otimes k\left(\sigma\left(x^{\prime}\right)\right) \rightarrow T_{X} \otimes k\left(x^{\prime}\right)$ contains $\operatorname{Im}\left(T_{\Sigma_{1}^{\prime}} \otimes k\left(x^{\prime}\right) \rightarrow T_{X} \otimes k\left(x^{\prime}\right)\right)$. This is true as long as $C_{1}$ passes through both $x$ and $x^{\prime}$. By deforming $C_{1}$ in $X$ passing through the fixed points $x$ and $x^{\prime}$, we get a family $T$ of $\Sigma_{1}$ 's passing through $x$ and $x^{\prime}$. The Zariski tangent space $T_{X} \otimes k\left(x^{\prime}\right)$ is generated by the images $\operatorname{Im}\left(T_{\Sigma_{1}^{\prime}} \otimes k\left(x^{\prime}\right) \rightarrow T_{X} \otimes k\left(x^{\prime}\right)\right)$ as $\Sigma_{1}$ runs through the family $T$. So $d \pi\left(\sigma\left(x^{\prime}\right)\right)$ is surjective and hence $\pi$ is étale at $\sigma\left(x^{\prime}\right)$. Since $x^{\prime} \in C$ is arbitrary, we know that $\pi$ is étale along $\sigma(C)$. As a result we have $\mathscr{N}_{\sigma(C) / Y} \cong \mathscr{N}_{C / X}$. It follows that the deformation of $\sigma(C)$ in $Y$ covers an open neighborhood of $[C]$ in $M^{0}$. Hence for a general $[C] \in M^{0}$ there is always a lifting of $C$ to $Y$ and $\pi$ is étale along the lifting (note that we don't require $x \in \Sigma_{C}$ anymore). Notations. By lifting $C \subset X$ to $Y$, we might get many unbalanced unbalanced components of very free rational curves on $Y$ of the same generic splitting type of the normal bundle. Let $M^{\prime}$ be one of these components such that $\pi$ is étale along $C^{\prime}$ for any $\left[C^{\prime}\right] \in M^{\prime}$. With respect to this $M^{\prime}$, we can do the same constructions on $Y$ as in the previous section. We use the notation $\Pi_{C^{\prime}}$ instead of $\Sigma_{C^{\prime}}$ for the surface constructed from a general point $\left[C^{\prime}\right] \in M^{\prime}$. Similarly we will use $\Pi^{\prime}$ and $\tilde{\Pi}$ instead of $\Sigma^{\prime}$ and $\tilde{\Sigma}$.

Step 2. For a general $\left[C^{\prime}\right] \in M^{\prime}$, the pair $C^{\prime} \subset \Pi_{C^{\prime}}^{\prime}$ is also equivalent to a conic on $\mathbb{P}^{2}$. Hence we also have the concept of pseudo-lines on $Y$.

Proof of step 2. Let $C \subset X$ be the image of $C^{\prime}$ via $\pi$. We know that $\pi$ is étale along $C^{\prime}$. The nearby deformations of $C^{\prime}$ in $Y$ with three points fixed induce the nearby deformations of $C$ in $X$ with the three image points fixed. Hence $\pi$ induces a morphism $\pi_{C^{\prime}}: \Pi_{C^{\prime}} \rightarrow \Sigma_{C}$ and $\pi_{C^{\prime}}^{\prime}: \Pi_{C^{\prime}}^{\prime} \rightarrow \Sigma_{C}^{\prime}$. For any point $y_{0} \in C^{\prime} \subset \Pi_{C^{\prime}}^{\prime}$, let $x_{0}=\pi_{C^{\prime}}^{\prime}\left(y_{0}\right) \in C \subset \Sigma_{C}^{\prime}$ be the image. Consider the following diagram

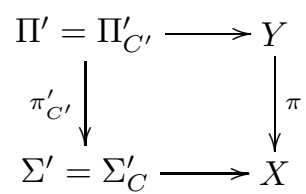

Since both $T_{\Pi^{\prime}, y_{0}} \otimes k\left(y_{0}\right) \rightarrow T_{Y, y_{0}} \otimes k\left(y_{0}\right)$ and $T_{\Sigma^{\prime}, x_{0}} \otimes k\left(x_{0}\right) \rightarrow T_{X, x_{0}} \otimes k\left(x_{0}\right)$ are injective. Together with the fact that $\pi$ is étale at $y_{0}$, we know $d \pi_{C^{\prime}}^{\prime}: T_{\Pi^{\prime}, y_{0}} \otimes k\left(y_{0}\right) \rightarrow$ $T_{\Sigma^{\prime}, x_{0}} \otimes k\left(x_{0}\right)$ is an isomorphism. Hence $\pi_{C^{\prime}}^{\prime}$ is étale along $C^{\prime}$. Hence $\left(\pi_{C^{\prime}}^{\prime}\right)^{-1}(C)$ is a disjoint union of $C^{\prime}$ with some other divisor $D^{\prime} \subset \Pi^{\prime}$. We already know that as a divisor, $C$ is nef and big on $\Sigma^{\prime}$. This implies that $\left(\pi_{C^{\prime}}^{\prime}\right)^{-1}(C)$ is also nef and big and hence connected. Thus we get $D^{\prime}=\emptyset$. Hence $\pi_{C^{\prime}}^{\prime}$ is finite of degree 1 , which means that it is isomorphism since $\Sigma^{\prime}$ is normal.

Step 3. The morphism $\pi$ maps a general pseudo-line on $Y$ to a pseudo-line on $X$.

Proof of step 3. Let $L^{\prime} \subset Y$ be a general pseudo-line on $Y$. Then by definition, there is some general point $\left[C^{\prime}\right] \in M^{\prime}$ such that $L^{\prime}$ is the image of a line $L_{1}^{\prime} \subset \Pi_{C^{\prime}}^{\prime}$. Since $\pi_{C^{\prime}}^{\prime}$ is an isomorphism, $L_{1}:=\pi_{C^{\prime}}^{\prime}\left(L_{1}^{\prime}\right)$ is a line on $\Sigma_{C}^{\prime}$, where $C \subset X$ is the image of $C^{\prime}$. Then $L=\pi\left(L^{\prime}\right)$, as the image of $L_{1} \subset \Sigma_{C}^{\prime}$, is a pseudo-line by definition.

Step 4. For a general pseudo-line $L \subset X, \pi^{-1}(L)$ is a disjoint union of $d$ pseudolines on $Y$. On $Y$, there is a unique pseudo-line connecting a general pair of points. 
Proof of step 4. It is easy to see from the definition of $Y$ that the degree $d=\operatorname{deg}(\pi)$ is the number of pseudo-lines connecting a general pair of points on $X$. Let $d^{\prime}$ be the number of pseudo-lines on $Y$ connecting a general pair of points. Let $(x, y) \in X \times X$ be a general pair of points on $X$ and $L_{1}, \ldots, L_{d}$ be the pseudo-lines connecting them. Let $\pi^{-1}(x)=\left\{x_{1}, \ldots, x_{d}\right\}$ and $\pi^{-1}(y)=\left\{y_{1}, \ldots, y_{d}\right\}$. There are $d^{\prime} d^{2}$ pseudo-lines $L_{i j k}^{\prime}$ connecting $x_{i}$ and $y_{j}$, where $i, j=1, \ldots, d$ and $k=1, \ldots, d^{\prime}$. Their images under $\pi$ are exactly the pseudo-lines $\left\{L_{i}\right\}$ connecting $x$ and $y$. On the other hand, the inverse image $\pi^{-1}\left(L_{i}\right)$ can contain at most $d$ pseudo-lines for the degree reason. It follows that $d^{\prime}=1$ and $\pi^{-1}\left(L_{i}\right)$ consists of $d$ pseudo-lines. These pseudo-lines are disjoint since $\pi$ is étale along any of them.

Proof of Proposition. From Step 4 and Lemma 3.9, we know that $C^{\prime} \subset Y$ is equivalent to a conic on $\mathbb{P}^{3}$ for general $\left[C^{\prime}\right] \in M^{\prime}$. Let $U \subset Y$ be the maximal open subset with an open immersion $\tilde{\rho}: U \rightarrow \mathbb{P}^{3}$ that realizes the above equivalence. Then a pseudo-line $L^{\prime} \subset U$ corresponds to a line on $\mathbb{P}^{3}$ and hence we will call $L^{\prime}$ a line instead of a pseudo-line. We already see that for a general pseudo-line $L \subset X, \pi$ is étale along $\pi^{-1}(L) \subset U$. Hence there is an open subscheme $V \subset X$ such that $\left.\pi\right|_{\pi^{-1}(V)}: \pi^{-1}(V) \rightarrow V$ is étale and $\pi^{-1}(V) \subset U$. Define $\rho=\left.\tilde{\rho}\right|_{\pi^{-1}(V)}$ then the proposition follows.

\subsection{Case II: Section of Hirzebruch surface.}

Situation 3.11. In this whole subsection we will assume Situation 3.1 with one further assumption that $C \subset \Sigma_{C}^{\prime}$ is equivalent to a positive section of a Hirzebruch surface $F_{n}$ for general $[C] \in M$.

Recall that by definition, there is a natural fibration $\pi_{n}: F_{n}=\mathbb{P}(\mathcal{O} \oplus \mathcal{O}(-n)) \rightarrow$ $\mathbb{P}^{1}$. By blowing up at smooth points, we may assume that the above equivalence is given by a morphism $\sigma: \tilde{\Sigma}=\tilde{\Sigma}_{C} \rightarrow F_{n}$, which is an isomorphism on a neighborhood of $C$ and the image of $C$ is a positive section of $\pi_{n}$. On $\tilde{\Sigma}$, there is a distinguished divisor $D$ that corresponds to the negative section of $F_{n}$ with $D^{2}=-n$. Note that $D$ need not be irreducible but there is a unique component $D_{h}$ which is a horizontal section. It is easy to see that $C$ can only meet $D$ at points of $D_{h}$ since $\tilde{\Sigma} \rightarrow F_{n}$ is blowing up centered away from $C$. Let $F \subset \tilde{\Sigma}$ be a general fiber of $\pi_{n} \circ \sigma: \tilde{\Sigma} \rightarrow \mathbb{P}^{1}$. Then $F$ is a smooth rational curve on $\tilde{\Sigma}$.

Definition 3.12. Let $\Gamma=\cup \Gamma_{i}$ be a nodal curve, and $Y$ be a smooth projective variety. Let $\varphi: \Gamma \rightarrow Y$ be a morphism such that $\varphi$ is an immersion on a neighborhood of each node and $d \varphi(x): T_{\Gamma} \otimes k(x) \rightarrow T_{Y} \otimes k(\varphi(x))$ is injective for all smooth point $x$ of $\Gamma$. We define the normal bundle of $\varphi$ to be

$$
\mathscr{N}_{\Gamma / Y}=\mathscr{N}_{\varphi}=\left[\operatorname{ker}\left(\varphi^{*} \Omega_{Y}^{1} \rightarrow \Omega_{\Gamma}^{1}\right)\right]^{\vee}
$$

Note that the above definition agrees with Definition 2.6 when $\Gamma$ is smooth. Since a nodal curve is always a local complete intersection, we know that $\mathscr{N}_{\Gamma / Y}$ is locally free. To better understand the normal bundle at the nodal points, let's assume that $\Gamma=\Gamma_{1} \cup \Gamma_{2}$ be a union to two smooth curves and let $p$ be the nodal point. We always have the following exact sequence, [12] and [34],

$$
\left.0 \longrightarrow \mathscr{N}_{\Gamma_{1} / Y} \longrightarrow \mathscr{N}_{\Gamma / Y}\right|_{\Gamma_{1}} \longrightarrow k(p) \longrightarrow 0
$$


This realizes $\left.\mathscr{N}_{\Gamma / Y}\right|_{\Gamma_{1}}$ as the sheaf of sections of $\mathscr{N}_{\Gamma_{1} / Y}$ that are either regular or have a simple pole at $p$ in the direction of $T_{\Gamma_{1}, p}$. A similar interpretation holds on $\Gamma_{2}$. The sheaf $\mathcal{T}:=\operatorname{Ext}_{\mathcal{O}_{\Gamma}}^{1}\left(\Omega_{\Gamma}, \mathcal{O}_{\Gamma}\right)$ is a torsion sheaf supported at $p$ whose fiber is canonically isomorphic to $\left.\mathcal{T}\right|_{p}=T_{\Gamma_{1}, p} \otimes T_{\Gamma_{2}, p}$. The natural quotient map $\mathscr{N}_{\Gamma / Y} \rightarrow \mathcal{T}$ induce an isomorphism $\left(\left.\mathscr{N}_{\Gamma / Y}\right|_{\Gamma_{1}}\right) / \mathscr{N}_{\Gamma_{1} / Y} \cong \mathcal{T}$. With the above preparation, we are ready to prove the following

LEMMA 3.13. Under Situation 3.11, the following does not happen: on $\tilde{\Sigma}$, the curve $C$ meets $D$ at least once and for a general fiber $F$ we have $\mathscr{N}_{F / X} \cong \mathcal{O} \oplus \mathcal{O}(1)$.

Proof. We prove the lemma by contradiction. So we assume the above situation happens. Since $C \cdot D \geq 1$, the curve $C$ degenerates to a general fiber $F$ and another positive section $C^{\prime}$. We have the following picture.

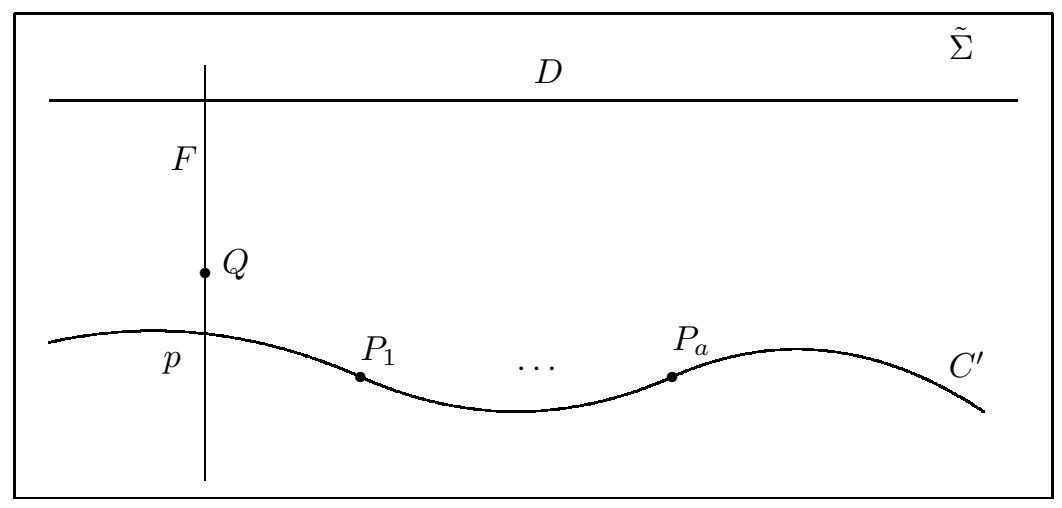

Since $\left(C^{\prime}\right)^{2}=(C-F)^{2}=C^{2}-2(C \cdot F)=b-2$, we have $\mathscr{N}_{C^{\prime} / \tilde{\Sigma}} \cong \mathcal{O}(b-2)$. Since $C \cdot D \geq 1$ and the tangent map $d \tilde{\phi}$ of $\tilde{\phi}: \tilde{\Sigma} \rightarrow X$ is injective along $C$, we know that $d \tilde{\phi}$ is also injective along a general fiber $F$. Hence we have the following short exact sequence

$$
\left.0 \longrightarrow \mathscr{N}_{F / \tilde{\Sigma}} \longrightarrow \mathscr{N}_{F / X} \longrightarrow \mathscr{N}_{\tilde{\Sigma} / X}\right|_{F} \longrightarrow 0
$$

It follows, from the above sequence and the assumption, that $\left.\mathscr{N}_{\tilde{\Sigma} / X}\right|_{F} \cong \mathcal{O}(1)$. Since $\left.\mathscr{N}_{\tilde{\Sigma} / X}\right|_{C} \cong \mathcal{O}(a)$, see Corollary 2.7 , we get that $\left.\mathscr{N}_{\tilde{\Sigma} / X}\right|_{C^{\prime}} \cong \mathcal{O}(a-1)$. Consider the following short exact sequence

$$
\left.0 \longrightarrow \mathscr{N}_{C^{\prime} / \tilde{\Sigma}} \longrightarrow \mathscr{N}_{C^{\prime} / X} \longrightarrow \mathscr{N}_{\tilde{\Sigma} / X}\right|_{C^{\prime}} \longrightarrow 0
$$

and we get $\mathscr{N}_{C^{\prime} / X} \cong \mathcal{O}(b-2) \oplus \mathcal{O}(a-1)$. Let $\Gamma=F \cup C^{\prime} \subset \tilde{\Sigma}$ and let $p$ be the nodal point. Consider the natural morphism $\varphi: \Gamma \rightarrow X$. The deformation problem of $\varphi$ with only the target $X$ being fixed is controlled by the cotangent complex of $\varphi$,

$$
L_{\varphi}^{*}:=\left\{0 \rightarrow \varphi^{*} \Omega_{X}^{1} \rightarrow \Omega_{\Gamma}^{1} \rightarrow 0\right\}
$$

Namely, the first order deformation is given by $\operatorname{Ext}_{\Gamma}^{1}\left(L_{\varphi}^{*}, \mathcal{O}_{\Gamma}\right)$ and the obstruction space is in $\operatorname{Ext}_{\Gamma}^{2}\left(L_{\varphi}^{*}, \mathcal{O}_{\Gamma}\right)$, see [26]. If we choose $F$ and $C^{\prime}$ general, then $d \varphi$ is injective at all smooth points of $\Gamma$ and $\varphi$ is an immersion on an open neighborhood of the node 
$p$. Hence we know that $L_{\varphi}^{*}$ is quasi-isomorphic to $\mathscr{N}_{\varphi}^{\vee}$ centered at degree -1 . Hence we have the following isomorphisms

$$
\operatorname{Ext}_{\Gamma}^{1}\left(L_{\varphi}^{*}, \mathcal{O}_{\Gamma}\right) \cong \mathrm{H}^{0}\left(\Gamma, \mathscr{N}_{\varphi}\right), \quad \operatorname{Ext}_{\Gamma}^{1}\left(L_{\varphi}^{*}, \mathcal{O}_{\Gamma}\right) \cong \mathrm{H}^{1}\left(\Gamma, \mathscr{N}_{\varphi}\right)
$$

We pick $Q \in F$ and $P_{1}, \ldots, P_{a} \in C^{\prime}$ to be general points. Consider the same deformation problem while we require the deformations to pass through the points $\left\{Q, P_{1}, \ldots, P_{a}\right\}$. Then the first order deformations and obstructions are given by $\mathrm{H}^{0}(\Gamma, \mathscr{E})$ and $\mathrm{H}^{1}(\Gamma, \mathscr{E})$ respectively, where $\mathscr{E}=\mathscr{N}_{\varphi}\left(-Q-\sum_{i=1}^{a} P_{i}\right)$. Since $F$ has trivial normal bundle in $\tilde{\Sigma}$, the $\mathcal{O}(1)$ direction in $\mathscr{N}_{F / X}$ is pointing outside $\tilde{\Sigma}$. Recall that $\left.\mathscr{E}\right|_{F}$ is the sheaf of sections of $\mathscr{N}_{F / X}(-Q)$ that are either regular or have a simple pole at $p$ along the direction of $T_{C^{\prime}, p}$. This shows that the restriction morphism

$$
\mathrm{H}^{0}\left(F,\left.\mathscr{E}\right|_{F}\right) \longrightarrow \mathscr{E} \otimes k(p)=\mathscr{N}_{\varphi} \otimes k(p)
$$

is surjective. In fact we have $\mathscr{N}_{F / X}(-Q) \cong \mathcal{O}(-1) \oplus \mathcal{O}$. The global section from the $\mathcal{O}$ factor and the rational section pointing to $T_{C^{\prime}, p}$ with a simple pole at $p$ form a basis for $\mathrm{H}^{0}\left(F,\left.\mathscr{E}\right|_{F}\right)$. They restrict to two linearly independent vectors in $\mathscr{N} \otimes k(p)$. To compute the cohomology groups of $\mathscr{E}$, we consider the following exact sequence.

$$
\left.\left.0 \longrightarrow \mathscr{E} \longrightarrow \mathscr{E}\right|_{F} \oplus \mathscr{E}\right|_{C^{\prime}} \longrightarrow \mathscr{E} \otimes k(p) \longrightarrow 0
$$

It follows easily from the interpretation of $\left.\mathscr{E}\right|_{F}$ and $\left.\mathscr{E}\right|_{C^{\prime}}$ that

$$
\mathrm{H}^{1}\left(F,\left.\mathscr{E}\right|_{F}\right)=0, \quad \mathrm{H}^{1}\left(C^{\prime},\left.\mathscr{E}\right|_{C^{\prime}}\right)=0
$$

and

$$
\operatorname{dim} \mathrm{H}^{0}\left(F,\left.\mathscr{E}\right|_{F}\right)=2 \quad \operatorname{dim} \mathrm{H}^{0}\left(C^{\prime},\left.\mathscr{E}\right|_{C^{\prime}}\right)=b-a
$$

Hence the long exact sequence associated to (6) becomes

$$
\begin{aligned}
& 0 \longrightarrow \mathrm{H}^{1}(\Gamma, \mathscr{E}) \longrightarrow \mathrm{H}^{0}\left(F,\left.\mathscr{E}\right|_{F}\right) \oplus \mathrm{H}^{0}\left(C^{\prime},\left.\mathscr{E}\right|_{C^{\prime}}\right) \stackrel{\alpha}{\longrightarrow} \mathscr{E} \otimes k(p) \\
& \longrightarrow \mathrm{H}^{1}(\Gamma, \mathscr{E}) \longrightarrow 0
\end{aligned}
$$

We already know that $\alpha$ is surjective. Hence we have

$$
\operatorname{dim} \mathrm{H}^{0}(\Gamma, \mathscr{E})=b-a, \quad \operatorname{dim} \mathrm{H}^{1}(\Gamma, \mathscr{E})=0
$$

So the deformation problem above is unobstructed. Note that the deformation that keeps the configuration $F \cup C^{\prime}$ is $(b-a-1)$-dimensional and hence a general deformation smooth out the node and gives a curve $\left[C_{1}\right] \in M^{0}$ passing through $\left\{Q, P_{1}, \ldots, P_{a}\right\}$. Now we deform $F \rightarrow X$ a little bit to get $F^{\prime} \cong \mathbb{P}^{1} \rightarrow X$ where the image of $F^{\prime}$ still passes through $p$ and $T_{F^{\prime}, p}$ is not contained in $T_{\Sigma^{\prime}, p}$. Then we get a morphism $\varphi^{\prime}: \Gamma^{\prime}=F^{\prime} \cup_{p^{\prime}} C^{\prime} \rightarrow X$. Pick $Q^{\prime} \in F^{\prime}$ we do the same deformation with respect to $\left\{Q^{\prime}, P_{1}, \ldots, P_{a}\right\}$. Since the vanishing of obstruction is an open condition, we know that this new deformation problem is still unobstructed which gives a different curve $\left[C_{2}\right] \in M^{0}$. From $C_{1}$ we get the surface $\Sigma_{1}=\Sigma$ and from $C_{2}$ we get a different surface $\Sigma_{2}$. Now since $C^{\prime}$ is component of the degeneration of both $C_{1}$ and 
$C_{2}$ with $a+1$ points fixed, $C^{\prime}$ lies on both of $\Sigma_{1}$ and $\Sigma_{2}$. Consider the deformation of $C^{\prime}$ in $X$ passing through $\left\{P_{1}, \ldots, P_{a}\right\}$. If we consider $C^{\prime}$ as a curve on $\Sigma_{1}^{\prime}$, and we can do the deformation of $C^{\prime}$ in $\Sigma_{1}^{\prime}$; Similarly we can also do the same deformation on $\Sigma_{2}^{\prime}$. As a result, the curve $C^{\prime}$ can move along both of the directions $T_{F, p}$ and $T_{F^{\prime}, p}$ at the point $p$. But this is impossible since $\mathscr{N}_{C^{\prime} / X}\left(-\sum_{i=1}^{a} P_{i}\right) \cong \mathcal{O}(-1) \oplus \mathcal{O}(b-a-2)$ is not globally generated.

The main result of this subsection is the following

Proposition 3.14. Assume that the anti-canonical divisor $-K_{X}$ is nef together with Situation 3.11, then for a general fiber $F \subset \tilde{\Sigma}$, the morphism $F \rightarrow X$ has at worst nodal image and $\mathscr{N}_{F / X}=\mathcal{O} \oplus \mathcal{O}$.

Proof. On $\tilde{\Sigma}$ we have the divisor class $C=D+c F$ for some integer $c$. We have the following basic relations

$$
\begin{gathered}
C^{2}=D^{2}+2 c(D \cdot F)=-n+2 c=b \quad \Rightarrow \quad c=\frac{b+n}{2} \\
C \cdot D=D^{2}+c=-n+c \geq 0 \quad \Rightarrow \quad c \geq n
\end{gathered}
$$

The above relations imply that $b \geq n$. We still use $K_{X}$ to denote the pullback of $K_{X}$ to $\tilde{\Sigma}$. From the following

$$
a+b+2=C \cdot\left(-K_{X}\right)=D \cdot\left(-K_{X}\right)+c F \cdot\left(-K_{X}\right)
$$

and the assumption that $-K_{X}$ is nef, we get

$$
F \cdot\left(-K_{X}\right) \leq \frac{a+b+2}{c}=\frac{2(a+b+2)}{b+n} \leq \frac{4 b}{b}=4 .
$$

By construction, a general $F$ passes through a general point of $X$ and hence $F$ is free. As a result, the intersection number $F \cdot\left(-K_{X}\right)$ can only be 2,3 or 4 . To prove the proposition, we only need to rule out the cases $F \cdot\left(-K_{X}\right)$ being 3 or 4 .

If $F \cdot\left(-K_{X}\right)=4$ then $n=0, b=a+2$ and $D \cdot\left(-K_{X}\right)=0$. In this case, the divisor $D$ is just the other ruling of $\mathbb{P}^{1} \times \mathbb{P}^{1}$ and hence a general member of the class of $D$ is a rational curve that passes through a general point of $X$ and hence is free. This implies that $D \cdot\left(-K_{X}\right) \geq 2$, which is a contradiction.

If $F \cdot\left(-K_{X}\right)=3$ then we first show that $C \cdot D \geq 1$. In fact, if $C \cdot D=0$, i.e. $D^{2}+c F \cdot D=0$, then $c=n$. From (7), we get $b=n$. Then in (9), we get

$$
3=F \cdot\left(-K_{X}\right) \leq \frac{2(a+b+2)}{b+n}=\frac{2(a+b+2)}{2 b}=\frac{a+b+2}{b} \leq 2 .
$$

Hence we get contradiction again. Recall that there is an open neighborhood $\tilde{U}$ of $C$ inside $\tilde{\Sigma}$ such that the morphism $\tilde{U} \rightarrow X$ has injective tangent map at each point. The fact that $C \cdot D \geq 1$ implies that $F \subset \tilde{U}$ for general $F$. This implies that $F \rightarrow X$ has injective tangent map at all points and hence $\mathscr{N}_{F / X} \cong \mathcal{O} \oplus \mathcal{O}(1)$, which is impossible by Lemma 3.13 .

3.3. Conclusion. Here we summarize the previous two subsections in the following theorem.

TheOREM 3.15. Let $X / \mathbb{C}$ be a smooth projective variety with $\operatorname{dim} X=3$. Let $M$ be an unbalanced component of very free rational curves such that for general $[C] \in M$, 
$C$ is a smooth rational curve on $X$ with normal bundle $\mathscr{N}_{C / X} \cong \mathcal{O}(a) \oplus \mathcal{O}(b)$, where $b-2 \geq a \geq 1$. Let $\Sigma$ be the surface swept out by deforming $C$ with $a+1$ points fixed as before. Let $\Sigma^{\prime}$ be its normalization. Then we have one of the following to cases.

Case I: The pair $C \subset \Sigma^{\prime}$ is equivalent to a conic in $\mathbb{P}^{2}$. In this case, there is a finite morphism $\pi: Y \rightarrow X$ and there is an open neighborhood $V \subset X$ of $C$ such that $\pi^{-1}(V) \rightarrow V$ is étale. Furthermore, there is an open immersion $\rho: \pi^{-1}(V) \rightarrow \mathbb{P}^{3}$ such that any lift $C \rightarrow \pi^{-1}(V)$ is a conic on $\mathbb{P}^{3}$.

Case II: The pair $C \subset \Sigma^{\prime}$ is equivalent to a positive section of a Hirzebruch surface $F_{n}$. In this case, if we further assume that $-K_{X}$ is nef then a general fiber $F$ of $\tilde{\Sigma}$ gives a free rational curve on $X$ with trivial normal bundle, i.e. $\mathscr{N}_{F / X} \cong \mathcal{O} \oplus \mathcal{O}$. Let $S$ be the component of the $\overline{\mathcal{M}}_{0,0}(X, \beta)$ that parameterizes such curves $F$. Then the natural morphism $\varphi: C \rightarrow S$ is a rational curve on $S$ that connects a general pair of points on $S$. In particular, $S$ is rationally connected and hence rational.

Proof. The theorem is pretty much the combination of Proposition 3.3, Proposition 3.10 and Proposition 3.14. We only need to show that $S$ is rationally connected in case II. Fix a general point $[F] \in S$, then there is some $C \subset \Sigma$ and $F \subset \Sigma$. Let $x$ be the point that $C$ meets $F$. By deforming $C$ passing through the fixed point $x$, we get a family of $\Sigma$ 's. The fiber of any such $\Sigma$ at the point $x$ is always the fixed $F$. Hence we get a covering family of rational curves on $S$ passing through the fixed point $[F]$. This means that $S$ is rationally connected.

Definition 3.16. Let $X$ and $M$ be as in the theorem. When Case I happens, we say that $M$ is an unbalanced component of conic type; when Case II happens, we say that $M$ is of fibration type.

Corollary 3.17. Let $X$ be a smooth projective threefold of Picard number 1. If $X$ has an unbalanced component $M$ of very free rational curves of conic type, then $X \cong \mathbb{P}^{3}$ and $M$ is the space of conics on $X$.

Proof. Let $\pi: Y \rightarrow X$ be the finite morphism we get from the theorem. Then $\pi$ is étale above $V \subset X$. Since $V$ contains a general curve $C$ and $X$ has Picard number 1, the complement of $V$ in $X$ has dimension less than or equal to 1 . Since $X$ is simply connected (this follows from the fact that it is rationally connected), we get that $V$ is also simply connected. This implies that $\operatorname{deg}(\pi)=1$ and hence $V=\pi^{-1}(V) \subset \mathbb{P}^{3}$. We have the identification of the Picard groups $\operatorname{Pic}(X)=\operatorname{Pic}(V)=\mathbb{Z} H$. From the fact that $\left.\mathcal{O}_{X}\left(-K_{X}\right)\right|_{V} \cong \Omega_{V}^{3}=\mathcal{O}_{V}(-4 H)$, we get $-K_{X} \cong 4 H$. So $X$ is Fano threefold of index 4 , which implies that $X \cong \mathbb{P}^{3}$.

4. Non-triviality of the Abel-Jacobi mapping. In this section, we use the technique of intermediate Jacobian and the Abel-Jacobi mapping to prove Theorem 1.4 .

4.1. Intermediate Jacobian and Abel-Jacobi mapping. The main reference to this section are [5], [36], [1], [2], [10] and [21].

Let $X$ be a smooth projective variety over $\mathbb{C}, \operatorname{dim} X=3$. We use $\mathrm{H}^{*}(X)$ to denote $\mathrm{H}^{*}(X, \mathbb{Z}) /$ torsion. We have the following Hodge decomposition.

$$
\mathrm{H}^{3}(X) \otimes \mathbb{C}=\mathrm{H}^{3,0}(X) \oplus \mathrm{H}^{2,1}(X) \oplus \mathrm{H}^{1,2}(X) \oplus \mathrm{H}^{0,3}(X)
$$

Let $W(X):=\mathrm{H}^{1,2}(X) \oplus \mathrm{H}^{0,3}(X)$ and let $U(X) \subset W(X)$ be the lattice defined by the image of $\mathrm{H}^{3}(X)$ under the projection. We define a Hermitian form on $W(X)$ by

$$
(\alpha, \beta)=h(\alpha, \beta):=2 i \int_{X} \alpha \wedge \bar{\beta}
$$


Then the imaginary part of $h$ restricts to an integral, unimodular, alternating form on $U(X)$.

Definition 4.1. Let $X$ be as above, the triple $(W(X), U(X), h)$ is called the intermediate Jacobian of $X$ and denoted by $J(X)$.

Proposition 4.2. ([5]) If $\mathrm{H}^{1}(X)=0$ and $\mathrm{H}^{0,3}(X)=0$ then $J(X)$ is a principally polarized abelian variety. In particular, if $X$ is Fano then $J(X)$ is a principally polarized abelian variety.

From now on, we always keep the assumption of the proposition above. The following proposition is well known, see [5] and [36].

Proposition 4.3. Let $X$ be a smooth projective threefold with $\mathrm{H}^{1}(X)=0$ and $\mathrm{H}^{0,3}(X)=0$, and $C \subset X$ be a smooth curve on $X$. Let $\tilde{X}$ be the blow-up of $X$ along the curve $C$, then we have canonical isomorphism

$$
J(\tilde{X}) \cong J(X) \oplus J(C)
$$

as principally polarized abelian varieties, where $J(C)$ is the jacobian of the curve.

We also need the following basic property on the behavior of the intermediate Jacobian under the operation of a flop.

Proposition 4.4. Let $X$ be a smooth projective threefold and let $\chi: X \rightarrow X^{+}$ be a flop of $(-2)$-curves. Then $J\left(X^{+}\right) \cong J(X)$ canonically.

Proof. By the definition of a flop, we have a diagram

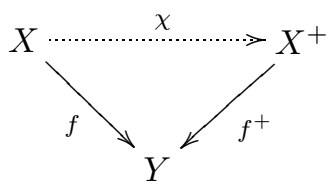

where both $f$ and $f^{+}$are small proper birational morphism. By a result of [30], $\chi$ is a composition of sequence of blow-ups and blow-downs centered along smooth rational curves. The proposition follows from the previous one.

Let

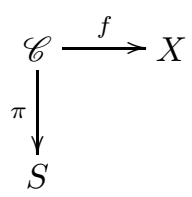

be a family of curves on $X$, i.e. $\mathscr{C}_{s}$ is a curve on $X$ for all $s \in S$. After fixing a general point $s_{0} \in S$, we get a map

$$
\Phi=\Phi_{S}: S \rightarrow J(X) .
$$

This is actually a morphism which induces

$$
\Psi=\Psi_{S}: \operatorname{Alb}(S) \rightarrow J(X) .
$$

We refer to [5] and [36] for more details. 
Definition 4.5. Both $\Phi$ and $\Psi$ are called the Abel-Jacobi mapping associate to the family $\mathscr{C} \rightarrow S$.

Now let's consider the infinitesimal version of the Abel-Jacobi mapping. Fix a smooth curve $C \subset X$, then we have the following exact sequence

$$
\left.0 \longrightarrow \mathscr{N}_{C / X}^{\vee} \longrightarrow \Omega_{X}^{1}\right|_{C} \longrightarrow \Omega_{C}^{1} \longrightarrow 0
$$

This induces an exact sequence as the following

$$
\left.0 \longrightarrow \wedge^{2}\left(\mathscr{N}_{C / X}^{\vee}\right) \longrightarrow \Omega_{X}^{2}\right|_{C} \longrightarrow \Omega_{C}^{1} \otimes \mathscr{N}_{C / X}^{\vee} \longrightarrow 0
$$

By taking the associated long exact sequence, we get an natural surjection

$$
\alpha: \mathrm{H}^{1}\left(C,\left.\Omega_{X}^{2}\right|_{C}\right) \longrightarrow \mathrm{H}^{1}\left(C, \Omega_{C}^{1} \otimes \mathscr{N}_{C / X}^{\vee}\right) \cong \mathrm{H}^{0}\left(C, \mathscr{N}_{C / X}\right)^{\vee}
$$

where the isomorphism is Serre duality. Note that if $\mathrm{H}^{1}\left(C, \wedge^{2} \mathscr{N}_{C / X}^{\vee}\right)=0$ then $\alpha$ is an isomorphism. Let

$$
r: \mathrm{H}^{1}\left(X, \Omega_{X}^{2}\right) \longrightarrow \mathrm{H}^{1}\left(C,\left.\Omega_{X}^{2}\right|_{C}\right)
$$

be the natural restriction map. Then the composition $\phi=\alpha \circ r$ is the dual of $d\left(\Phi_{S}\right)$ and the point $[C]$ when $\mathscr{C} \rightarrow S$ is the universal family. We call $\phi$ the infinitesimal Abel-Jacobi mapping.

Proposition 4.6. ([37] Lemma 2.8) Suppose X can be embedded in a smooth 4 dimensional variety $W$. Then there is a commutative diagram as following

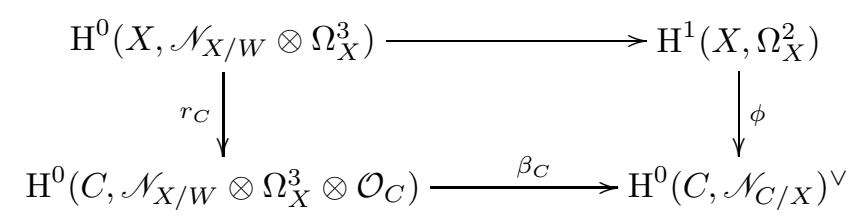

Here the map $\beta_{C}$ fits into the following long exact sequence

$$
\begin{aligned}
& \mathrm{H}^{0}\left(C, \mathscr{N}_{X / W} \otimes \Omega_{X}^{3} \otimes \mathcal{O}_{C}\right) \longrightarrow \beta_{C} \mathrm{H}^{0}\left(C, \mathscr{N}_{C / X}\right)^{\vee} \longrightarrow \\
& \quad \rightarrow \mathrm{H}^{1}\left(C, \mathscr{N}_{C / W} \otimes \Omega_{X}^{3}\right) \longrightarrow \mathrm{H}^{1}\left(C, \mathscr{N}_{X / W} \otimes \Omega_{X}^{3} \otimes \mathcal{O}_{C}\right) \longrightarrow 0
\end{aligned}
$$

COROLlary 4.7. Notations and assumptions as above, if $\mathscr{N}_{C / X} \cong \mathcal{O} \oplus \mathcal{O}$ and the following two conditions hold, then the infinitesimal Abel-Jacobi mapping $\phi$ is nontrivial.

(1) The restriction map $r_{C}: \mathrm{H}^{0}\left(X, \mathscr{N}_{X / W} \otimes \Omega_{X}^{3}\right) \rightarrow \mathrm{H}^{0}\left(C, \mathscr{N}_{X / W} \otimes \Omega_{X}^{3} \otimes \mathcal{O}_{C}\right)$ is surjective;

(2) $h^{1}\left(C, \mathscr{N}_{C / W} \otimes \Omega_{X}^{3}\right)-h^{1}\left(C, \mathscr{N}_{X / W} \otimes \Omega_{X}^{3} \otimes \mathcal{O}_{C}\right) \leq 1$. 
4.2. Nontriviality of Abel-Jacobi mapping. To prove the main result of this section, we need a description of double covers. Let $\pi: X \rightarrow V$ be a double cover between smooth algebraic varieties, $R \subset X$ be the ramification locus and $B \subset V$ be the image of $R$. Then $R \cong B$ are smooth and there is a line bundle $\mathscr{L}$ on $V$ such that $\mathscr{L}^{\otimes 2} \cong \mathcal{O}_{V}(B)$. There is a section $\sigma \in \Gamma\left(V, \mathscr{L}^{\otimes 2}\right)$ such that $B=\operatorname{div}(\sigma)$. We have the following diagram

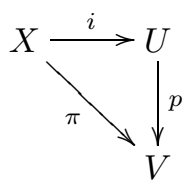

where $U=\operatorname{Spec}_{V}\left(\operatorname{Sym}^{*}\left(\mathscr{L}^{-1}\right)\right)$ is the space of $\mathscr{L}$. On $U$, there is a canonical section $y \in \Gamma\left(U, p^{*} \mathscr{L}\right)$ and $X=\operatorname{div}\left(y^{2}-p^{*} \sigma\right)$. It is easy to see that $T_{U / V}=p^{*} \mathscr{L}$ and hence we have the following exact sequence

$$
0 \longrightarrow p^{*} \mathscr{L} \longrightarrow T_{U} \longrightarrow p^{*} T_{V} \longrightarrow 0 .
$$

Then it is easy to see that $\mathscr{N}_{X / U} \cong p^{*} \mathscr{L}^{\otimes 2}$ and $\omega_{X} \cong \pi^{*}\left(\omega_{V} \otimes \mathscr{L}\right)$.

Lemma 4.8. Assume that $Q \subset \mathbb{P}^{n}, n \geq 4$, is a quadric hypersurface. Let $C \subset Q$ be a smooth conic rational curve in the smooth locus of $Q$. Let $\Pi=\Pi(C)$ be the plane spanned by $C$. Then $\mathscr{N}_{C / Q}$ has a direct summand of $\mathcal{O}_{\mathbb{P}^{1}}(4)$ if and only if $Q$ contains $\Pi$.

Proof. Consider the following short exact sequence

$$
\left.0 \longrightarrow \mathscr{N}_{C / Q} \longrightarrow \mathscr{N}_{C / \mathbb{P}^{n}} \longrightarrow \mathscr{N}_{Q / \mathbb{P}^{n}}\right|_{C} \longrightarrow 0
$$

It is easy to see that $\mathscr{N}_{C / \mathbb{P}^{n}} \cong \mathcal{O}(4) \oplus \mathcal{O}(2)^{\oplus(n-2)}$ and the $\mathcal{O}(4)$ summand is canonically isomorphic to $\mathscr{N}_{C / \Pi}$. If $\mathscr{N}_{C / Q}$ contains an $\mathcal{O}(4)$ summand, then this summand has to map isomorphically onto the $\mathscr{N}_{C / \Pi}$ summand of $\mathscr{N}_{C / \mathbb{P}^{n}}$. This means that $\Pi$ is tangent to $Q$ along $C$. This can happen only if $\Pi \subset Q$. The other direction is easy. $\square$

With the above preparations, we are ready to prove the following

Theorem 4.9. Let $X$ be a Fano threefold of index 1 or 2 and of Picard number 1. Assume that the intermediate Jacobian $J(X)$ is not zero. Let $S$ be a component of rational curves on $X$ with trivial normal bundle. Then the Abel-Jacobi mapping

$$
\Phi: S \rightarrow J(X)
$$

is nontrivial. In particular, $S$ is not rational.

Remark. The intermediate Jacobian $J(X)$ is trivial only when $X=X_{5}$ is of index 2 and degree 5 or when $X=X_{22}$ is of index 1 and genus 12 .

Proof. We prove the theorem case by case. We use $C$ to denote a general member of the family $S$.

First we consider the case when the index of $X$ is 1 and in this case the rational curves with trivial normal bundle are conics on $X$. Recall that for those of high genus, we use the method of "double projection from a line" and get the following diagram, 
see [20], [18], [19] and [7].

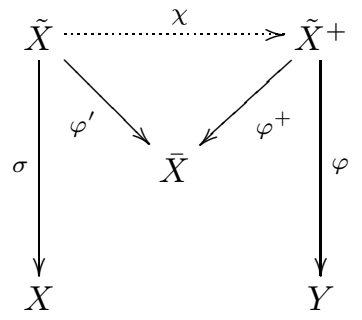

Recall that $\sigma$ is blow-up along a line, $\chi$ is a flop of $(-2)$-curves and $\varphi$ is an extremal contraction. Let $Z \subset S$ be the curve that parameterizes a component of the conics which meet $l$ where $l$ is a line on $X$ and the center of the blow-up $\sigma$. Such $Z$ always exists since we can pick $l$ general. Let $\mathscr{C}_{Z} \rightarrow Z$ be the family over $Z$. After blowing up and the flop, this gives a family $\mathscr{C}_{Z}^{+} \rightarrow Z$ of rational curves on $\tilde{X}^{+}$. Since $\chi$ is a flop of $(-2)$-curves, by Proposition 4.1, we know that there is a canonical isomorphism $J(X) \cong J\left(\tilde{X}^{+}\right)$and we get the following commutative diagram

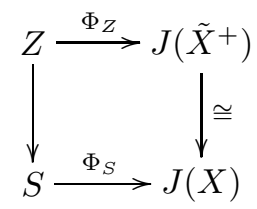

Note that all curve in the family $\mathscr{C}_{Z}^{+} \rightarrow Z$ are contracted by $\varphi$ and if we can show that $\Phi_{Z}$ is nontrivial then $\Phi_{S}$ is also nontrivial.

$\mathbf{g}=10$ : In this case, $\varphi: \tilde{X}^{+} \rightarrow Y$ blows down a divisor onto a smooth curve of genus 2 on $Y \cong Q \subset \mathbb{P}^{4}$. This implies that $Z$ is of genus 2 and $J\left(\tilde{X}^{+}\right) \cong J(Z)$ and hence $\Phi_{Z}$ is nontrivial.

g=9: In this case, $\varphi: \tilde{X}^{+} \rightarrow Y$ blows down a divisor onto a smooth curve of genus 3 on $Y \cong \mathbb{P}^{3}$. This implies that $Z$ is of genus 3 and $J\left(\tilde{X}^{+}\right) \cong J(Z)$ and hence $\Phi_{Z}$ is nontrivial.

$\mathbf{g}=8$ : In this case $\varphi: \tilde{X}^{+} \rightarrow Y$ is a standard conic bundle over $Y \cong \mathbb{P}^{2}$ with discriminant $\Delta \subset \mathbb{P}^{2}$ being of degree 5 . In this case $J\left(\tilde{X}^{+}\right)$is the prim variety $\operatorname{Pr}(\tilde{\Delta} / \Delta)$ of the double cover $\tilde{\Delta} \rightarrow \Delta$, see [10] and [1]. Then $Z \rightarrow \Delta_{0}$ is a double cover of a component $\Delta_{0}$ of $\Delta$. If $\operatorname{deg} \Delta_{0}=1$ then $Z$ is an elliptic curve; If $\operatorname{deg}\left(\Delta_{0}\right)=2$ then $Z$ has genus 2 ; If $\operatorname{deg}\left(\Delta_{0}\right)=3$ then $Z$ has genus 2 or 3 , depending on whether $\Delta_{0}$ has a node or not; If $\operatorname{deg}\left(\Delta_{0}\right)=4$, then $Z$ has genus $7,6,5$ or 4 , depending on the number of nodes of $\Delta_{0}$; If $\Delta_{0}=\Delta$ then $Z \cong \tilde{\Delta}$. In any of the above cases, it is easy to check that the morphism $\Phi_{Z}$ is nontrivial. For example, if $\operatorname{deg}\left(\Delta_{0}\right)=1$ then the double cover $Z \rightarrow \Delta_{0}$ ramifies at 4 points and $\operatorname{Pr}\left(Z / \Delta_{0}\right)=J(Z)$ gives a factor of the $J(X) \cong \operatorname{Pr}(\tilde{\Delta} / \Delta)$. The Abel-Jacobi mapping $\Phi_{Z}$ maps $Z$ nontrivially to the factor $\operatorname{Pr}\left(Z / \Delta_{0}\right)$. The other cases are similar.

$\mathbf{g}=7$ : In [17] (Proposition 2.2), it is proved that $S \cong \Gamma^{(2)}$ the symmetric product to a smooth curve $\Gamma$ of genus 7 . It is also known that the intermediate Jacobian of $X$ is isomorphic to the Jacobian of $\Gamma$. Hence $\Phi_{S}$ is nontrivial.

For the remaining cases, we will use Corollary 4.7 to show the nontriviality of Abel-Jacobi mapping. We refer to the conditions in Corollary 4.7 as condition (1) and condition (2). 
$\mathbf{g}=6$ : In this case, $X$ is either (i)a section of the Grassmannian $G r(2,5)$ embedded by Plücker into $\mathbb{P}^{9}$ by a linear $\mathbb{P}^{7}$ and a quadric or (ii)the section by a quadric of a cone $\tilde{V}_{5} \subset \mathbb{P}^{7}$ over $V_{5} \subset \mathbb{P}^{6}$ where $V_{5}$ is a Fano threefold of Picard number 1 , index 2 and degree 5 , see $[20] \S 5.1$.

In case (i), we take

$$
C \subset X \subset W=G(2,5) \cap \mathbb{P}^{7}=G(2,5) \cap H_{1} \cap H_{2}
$$

Then we have $\mathscr{N}_{X / W} \cong \mathcal{O}_{X}(2 H)$ and $\Omega_{X}^{3} \cong \mathcal{O}(-H)$. Consider the following natural commutative diagram.

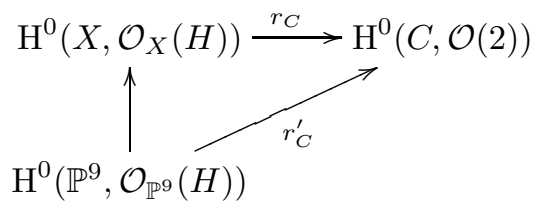

where $r_{C}^{\prime}$ is surjective. This implies that

$$
r_{C}: \mathrm{H}^{0}\left(X, \mathscr{N}_{X / W} \otimes \Omega_{X}^{3}\right) \rightarrow \mathrm{H}^{0}\left(C, \mathscr{N}_{X / W} \otimes \Omega_{X}^{3} \otimes \mathcal{O}_{C}\right) \cong \mathrm{H}^{0}(C, \mathcal{O}(2))
$$

is surjective and hence condition (1) holds. Set $G=G(2,5)$, then we have the following

$$
\left.0 \longrightarrow \mathscr{N}_{C / X} \longrightarrow \mathscr{N}_{C / W} \longrightarrow \mathscr{N}_{X / W}\right|_{C} \longrightarrow 0
$$

Note that $\left.\mathscr{N}_{X / W}\right|_{C} \cong \mathcal{O}(4)$ and $\mathscr{N}_{C / X} \cong \mathcal{O} \oplus \mathcal{O}$. Then it is easy to see that if $\mathscr{N}_{C / W}$ does not have a summand $\mathcal{O}(4)$ then condition (2) also holds and hence we know that the Abel-Jacobi mapping is nontrivial. So we only need to prove that $\mathscr{N}_{C / W}$ can not have a summand of $\mathcal{O}(4)$. We prove this by contradiction. Assume that $\mathscr{N}_{C / W} \cong \mathcal{O}(4) \oplus \mathcal{O}(2)^{\oplus 2}$. It is well known that $G=G r(2,5) \subset \mathbb{P}^{2}$ is cut out by quadrics, see [13]. Suppose $Q$ is a quadric hypersurface of $\mathbb{P}^{9}$ that contains $G$. Since $\mathscr{N}_{C / G}$ injects into $\mathscr{N}_{C / Q}$, we know that if $\mathscr{N}_{C / G}$ has an $\mathcal{O}(4)$ summand then so does $\mathscr{N}_{C / Q}$. By Lemma 4.8, we have $\Pi=\Pi(C) \subset Q$, where $\Pi(C)$ is the plane spanned by $C$. Since $Q$ is arbitrary, one sees that $\mathscr{N}_{C / G}$ contains an $\mathcal{O}(4)$ summand if and only if the plane $\Pi(C)$ is contained in $G$. From the following exact sequence

$$
0 \longrightarrow \mathscr{N}_{C / W} \longrightarrow \mathscr{N}_{C / G} \longrightarrow \mathcal{O}(2)^{\oplus 2} \longrightarrow 0
$$

one sees easily that if $\mathscr{N}_{C / W} \cong \mathcal{O}^{\oplus 2} \oplus \mathcal{O}(4)$, then $\mathscr{N}_{C / G}$ will have an $\mathcal{O}(4)$ summand. As a result, for a general conic $C$ on $X$ we have $\Pi(C) \subset G$ and hence $\Pi(C) \subset W$. This means that $W$ has a 2-dimensional family of planes. However, it is known that the planes on $W$ form a 1-dimensional family, see [27] (3.2).

In case (ii), the projection from the node of $\tilde{V}_{5}$ realizes $X$ as a double cover of $V_{5}$ that ramifies along a smooth divisor $B \in|2 H|$. Use the notations above for double covers, we take $W=U$ and then we have $\mathscr{N}_{X / W} \cong \mathcal{O}_{X}(2 H)$ and $\Omega_{X}^{3} \cong \mathcal{O}_{X}(-H)$. This gives the surjection condition (1) as before. To verify the condition (2), consider the following exact sequence.

$$
0 \longrightarrow \mathscr{N}_{C / X} \longrightarrow \mathscr{N}_{C / V_{5}} \longrightarrow \mathfrak{Q} \longrightarrow 0
$$

Note that $\mathscr{N}_{C / X} \cong \mathcal{O} \oplus \mathcal{O}$. The cokernel $\mathfrak{Q}$ is a skyscraper sheaf of degree 2 since $C \cdot R=2$, where $R$ is the ramification divisor. Then we get $\mathscr{N}_{C / V_{5}} \cong \mathcal{O} \oplus \mathcal{O}(2)$ or 
$\mathcal{O}(1) \oplus \mathcal{O}(1)$. We also have the following short exact sequence

$$
\left.0 \longrightarrow T_{W / V_{5}}\right|_{C} \longrightarrow \mathscr{N}_{C / W} \longrightarrow \mathscr{N}_{C / V_{5}} \longrightarrow 0
$$

Since $T_{W / V_{5}} \cong \pi^{*} \mathcal{O}_{V_{5}}(1)$, we have $\left.T_{W / V_{5}}\right|_{C} \cong \mathcal{O}(2)$. Then the sequence shows that $\mathscr{N}_{C / W} \cong \mathcal{O} \oplus \mathcal{O}(2)^{\oplus 2}$ or $\mathcal{O}(1)^{\oplus 2} \oplus \mathcal{O}(2)$. Condition (2) holds in either case.

$\mathbf{g}=5: \quad X=Q_{1} \cap Q_{2} \cap Q_{3} \subset \mathbb{P}^{6}$ is a complete intersection of 3 quadrics. We take $W=Q_{i} \cap Q_{j}$ where $1 \leq i \leq j \leq 3$. Then we have $\mathscr{N}_{X / W} \cong \mathcal{O}_{X}(2 H)$ and $\Omega_{X}^{3} \cong \mathcal{O}_{X}(-H)$. Condition (1) is readily verified. For condition (2), we consider

$$
\left.0 \longrightarrow \mathscr{N}_{C / X} \cong \mathcal{O}^{\oplus 2} \longrightarrow \mathscr{N}_{C / W} \longrightarrow \mathscr{N}_{X / W}\right|_{C} \cong \mathcal{O}(4) \longrightarrow 0
$$

From this one sees that condition (2) holds if $\mathscr{N}_{C / W}$ does not have a summand of $\mathcal{O}(4)$. Now suppose that $\mathscr{N}_{C / W}$ has an $\mathcal{O}(4)$ summand for all possible choice of $W$, then the sequence

$$
0 \longrightarrow \mathscr{N}_{C / W} \longrightarrow \mathscr{N}_{C / Q_{i}} \longrightarrow \mathcal{O}(4) \longrightarrow 0
$$

implies that $\mathscr{N}_{C / Q_{i}}$ also has a summand of $\mathcal{O}(4)$. By Lemma 4.8 , the plane $\Pi(C)$ is contained in $Q_{i}$. This is true for all $i=1,2,3$. Then $X$ should contain a linear $\mathbb{P}^{2}$. This is impossible because by adjunction formula, any smooth surface on $X$ is either $K 3$ or of general type.

$\mathbf{g}=4: X=Q \cap Y \subset \mathbb{P}^{5}$ is a complete intersection of a quadric and a cubic. Let's take $W=Q$ to be the quadric. We have $\mathscr{N}_{X / W} \cong \mathcal{O}_{X}(3 H)$ and $\Omega_{X}^{3} \cong \mathcal{O}_{X}(-H)$. We can verify condition (1) easily. Consider the exact sequence

$$
0 \longrightarrow \mathscr{N}_{C / X} \cong \mathcal{O}^{\oplus 2} \longrightarrow \mathscr{N}_{C / W} \longrightarrow \mathcal{O}(6) \longrightarrow 0
$$

We easily see that condition (2) holds as long as $\mathscr{N}_{C / W} ¥ \mathcal{O}^{\oplus 2} \oplus \mathcal{O}(6)$. On the other hand we have

$$
0 \longrightarrow \mathscr{N}_{C / W} \longrightarrow \mathscr{N}_{C / \mathbb{P}^{5}} \cong \mathcal{O}(2)^{\oplus 3} \oplus \mathcal{O}(4) \longrightarrow \mathcal{O}(4) \longrightarrow 0
$$

and this implies that $\mathscr{N}_{C / W}$ can not have a summand of degree greater than 4 . Hence condition (2) holds.

$\mathbf{g}=3: \quad X=X_{4} \subset \mathbb{P}^{4}$ and we take $W=\mathbb{P}^{4}$. We have $\mathscr{N}_{X / W}=\mathcal{O}(4 H)$ and $\Omega_{X}^{3} \cong \mathcal{O}_{X}(-H)$ and condition (1) follows easily. Condition (2) also easily follows from the fact that $\mathscr{N}_{C / W} \cong \mathcal{O}(2)^{\oplus 2} \oplus \mathcal{O}(4)$.

g=3: $X \rightarrow Q \subset \mathbb{P}^{4}$ is a double cover of a quadric threefold that ramifies along a surface $B$ of degree 8 . With the notations for double covers, we take $W=U$ and here $V=Q$ and $\mathscr{L}=\mathcal{O}_{V}(2 H)$. The we easily get $\Omega_{X}^{3} \cong \pi^{*} \mathcal{O}_{V}(-H)$ and $\mathscr{N}_{X / W} \cong \pi^{*} \mathcal{O}_{V}(4 H)$. Condition (1) is again easy to verify. For condition (2), we consider the following

$$
\left.0 \longrightarrow T_{W / V}\right|_{C} \cong \mathcal{O}(4) \longrightarrow \mathscr{N}_{C / W} \longrightarrow \mathscr{N}_{C / V} \longrightarrow 0
$$

On the quadric threefold $V$ we always have $\mathscr{N}_{C / V} \cong \mathcal{O}(2) \oplus \mathcal{O}(2)$ and hence $\mathscr{N}_{C / W} \cong$ $\mathcal{O}(4) \oplus \mathcal{O}(2) \oplus \mathcal{O}(2)$. Thus the condition (2) holds. 
$\mathbf{g}=\mathbf{2}: \quad X \rightarrow \mathbb{P}^{3}$ is double cover of $\mathbb{P}^{3}$ which ramifies along a smooth surface of degree 6. Take $W=U, V=\mathbb{P}^{3}$ and we get $\Omega_{X}^{3} \cong \pi^{*} \mathcal{O}_{V}(-H)$ and $\mathscr{N}_{X / W} \cong$ $\pi^{*} \mathcal{O}_{V}(6 H)$. Hence condition (1) holds. The exact sequence

$$
\left.0 \longrightarrow T_{W / V}\right|_{C} \cong \mathcal{O}(6) \longrightarrow \mathscr{N}_{C / W} \longrightarrow \mathscr{N}_{C / V} \cong \mathcal{O}(2) \oplus \mathcal{O}(4) \longrightarrow 0
$$

shows that $\mathscr{N}_{C / W} \cong \mathcal{O}(6) \oplus \mathcal{O}(4) \oplus \mathcal{O}(2)$. Thus condition (2) also holds.

Now we consider the cases when the index of $X$ is 2 . We prove case by case according to the $d=H^{3}$. Note that in this case, the curve $C$ is a line on $X$. We still use Corollary 4.7 to show nontriviality of Abel-Jacobi mapping.

$\mathbf{d}=4: X=Q_{1} \cap Q_{2} \subset \mathbb{P}^{5}$ is a complete intersection of two quadrics in $\mathbb{P}^{5}$. Take $W=Q_{1}$ and we have $\mathscr{N}_{X / W} \cong \mathcal{O}_{X}(2 H)$ and $\Omega_{X}^{3} \cong \mathcal{O}_{X}(-2 H)$. It is still easy to verify condition (1). We have the following two short exact sequences

$$
0 \longrightarrow \mathscr{N}_{C / X} \cong \mathcal{O} \oplus \mathcal{O} \longrightarrow \mathscr{N}_{C / W} \longrightarrow \mathcal{O}(2) \longrightarrow 0
$$

and

$$
0 \longrightarrow \mathscr{N}_{C / W} \longrightarrow \mathscr{N}_{C / \mathbb{P}^{5}} \cong \mathcal{O}(1)^{4} \longrightarrow \mathcal{O}(2) \longrightarrow 0
$$

It follows easily that $\mathscr{N}_{C / W} \cong \mathcal{O}(1)^{2} \oplus \mathcal{O}$. Hence condition (2) holds.

$\mathbf{d}=\mathbf{3}: X$ is a smooth cubic threefold. This case is well known, see [5].

$\mathbf{d = 2}: X \rightarrow \mathbb{P}^{3}$ is a double cover of $\mathbb{P}^{3}$ that ramifies along a smooth surface of degree 4. This case is studied in [37], Proposition (2.13).

$\mathbf{d}=\mathbf{1}: X$ is a smooth hypersurface of degree 6 in the weighted projective space $\mathbb{P}=\mathbb{P}(3,2,1,1,1)$ with weighted homogeneous coordinates $\left(x_{0}, x_{1}, x_{2}, x_{3}, x_{4}\right)$. Since $X$ is smooth, it must be contained inside the smooth locus of $\mathbb{P}$. Let $p r: \mathbb{P} \rightarrow \mathbb{P}^{2}$ be the projection to the last three coordinates. Let $C \subset X$ be a general line on $X$. Consider

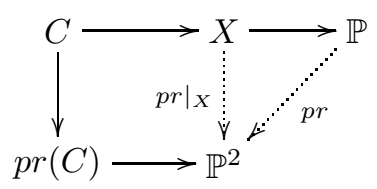

This shows that the homomorphisms

$$
\mathrm{H}^{0}\left(\mathbb{P}, \mathcal{O}_{\mathbb{P}}(n)\right) \longrightarrow \mathrm{H}^{0}(C, \mathcal{O}(n))
$$

is surjective for all $n \geq 0$, if $\operatorname{pr}(C)$ is not a single point. But it is clear that $\operatorname{pr}(C)$ is not a single point for general $C$. Otherwise, a general fiber of $\left.p r\right|_{X}$ always contains a line and hence reducible. But this is impossible by Bertini's theorem. Now we take $W=\mathbb{P}$ and we have $\mathscr{N}_{X / W} \cong \mathcal{O}_{X}(6)$ and $\Omega_{X}^{3} \cong \mathcal{O}_{X}(-2)$. Then the above surjection implies condition (1). For condition (2), note that $C$ is a line in the smooth locus of $\mathbb{P}$ and $\operatorname{pr}(C) \neq p t$, we know that $\mathscr{N}_{C / W}$ is ample. Hence $\mathrm{H}^{1}\left(C, \mathscr{N}_{C / W} \otimes \Omega_{X}^{3}\right)=0$, which implies condition (2).

4.3. Proof of main theorem. Now we are ready to prove the main theorem of this article.

TheOREm 4.10. Let $X$ be a Fano threefold of Picard number 1. If $X$ has an unbalanced component $M$ of very free rational curves, then $X=\mathbb{P}^{3}$ and $M$ is the space of conics on $X$. 
Proof. If $M$ is of conic type, then by Corollary 3.17 , we know that $X$ is $\mathbb{P}^{3}$ and $M$ is the space of conics on $X$. If $M$ is of fibration type, then by Theorem 3.15, a component $S$ of the space of rational curves with trivial normal bundle is rational. For index 3 and 4 cases, there is no such rational curves on $X$. For index 1 and 2 cases, the non-triviality of the associated Abel-Jacobi mapping implies that $S$ is not rational unless $X=X_{5}$ or $X=X_{22}$.

When $X=X_{5}$, let $S$ be the space of lines on $X$. Then by [11], we know $S=\mathbb{P}^{2}$ with the universal family being a projective bundle $\mathbb{P}(\mathscr{E})$ over $S$ and the $(-1,1)$-curves corresponds to a conic curve $\Delta \subset S$. Consider the universal family

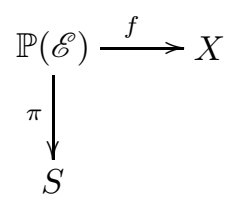

The morphism $f$ ramifies along $\pi^{-1}(\Delta)$. Let $B=f\left(\pi^{-1}(\Delta)\right)$. Now let $[C] \in M$ be a general point. Then by constructing the surface $\Sigma$ associated to $C$, we get a family of lines $\Sigma^{\prime} \rightarrow \mathbb{P}^{1}$ with a section $\sigma$. This gives a morphism $\varphi: \mathbb{P}^{1} \rightarrow S$ which has a lift $\sigma^{\prime}: \mathbb{P}^{1} \rightarrow \mathbb{P}(\mathscr{E})$.

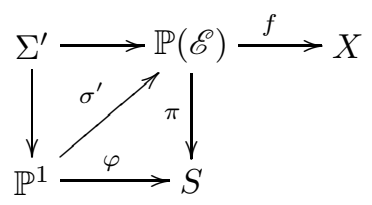

Where $f \circ \sigma^{\prime}$ gives the curve $C$. Since $\sigma^{\prime}\left(\mathbb{P}^{1}\right)$ meets $\pi^{-1}(\Delta)$, the curve $C$ is always tangent to $B$. This is impossible since $C$ is a general point in a component of very free rational curves.

The case $X=X_{22}$ can be ruled out similarly. In this case we also have $S \cong \mathbb{P}^{2}$ and the only difference is that $f$ ramifies along $\pi^{-1}(\Delta)$ where $\Delta$ is a degree 4 divisor on $S$. See the appendix for details.

\section{Appendix A. Space of conics on $X_{22}$.}

A.1. We work over the field $\mathbb{C}$ of complex numbers. Let $\mathscr{E}$ be a vector bundle on a variety $Z$, then we use $G(k, \mathscr{E})$ to denote the scheme that parameterizes $k$ dimensional fiberwise subspaces of $\mathscr{E}$. Hence $G(k, \mathscr{E})$ is a Grassmannian bundle over $Z$. When $\mathrm{k}=1$, it can also be written as $\mathbb{P}\left(\mathscr{E}^{*}\right)$. We similarly define $G\left(k_{1}, k_{2}, \ldots, k_{r}, \mathscr{E}\right)$, $0<k_{1}<\cdots<k_{r}<\operatorname{rank}(\mathscr{E})$, to be the relative Flag variety over $Z$.

A.2. In the whole article, we fix $X=X_{22} \subset \mathbb{P}^{13}$ to be a prime Fano threefold of genus 12. In particular, this means that $X$ is a smooth projective variety whose anti-canonical class $-K_{X}$ is very ample and generates $\operatorname{Pic}(X) \cong \mathbb{Z}$. The embedding $X \subset \mathbb{P}^{13}$ is given by the complete linear system $\left|-K_{X}\right|$ and the intersection of two general hyperplane sections gives a canonical curve of genus 12 . To better understand the structure of $X$, we introduce several notations. Let $V$ be a vector space. A net of alternating forms on $V$ is a surjective homomorphism $\eta: \wedge^{2} V \rightarrow N$ with $\operatorname{dim} N=3$. We use $G(k, V ; \eta)$ to denote $\left\{E \in G(k, V): \eta\left(\wedge^{2} E\right)=0\right\}$. We have the following structure theorem. 
Theorem A.3. (Mukai [28]) Let $X=X_{22} \subset \mathbb{P}^{13}$ be a prime Fano threefold of genus 12. Then there is a 7 dimensional vector space $V$ and a net of alternating forms, $\eta: \wedge^{2} V \rightarrow N$, such that $X=G(3, V ; \eta)$. Conversely, for a general such $\eta$, the variety $X=G(3, V ; \eta)$ is prime Fano threefold of genus 12 .

A.4. From now on, we fix a 7 dimensional vector space $V$ and a net of alternating forms $\eta$ as above such that $X=G(3, V ; \eta)$ is a Fano threefold of genus 12 . We use $\mathscr{E}_{3}$ to denote the canonical rank 3 subbundle of the trivial bundle $V \otimes \mathcal{O}_{X}$. Let $C \cong \mathbb{P}^{1} \subset X$ be a conic on $X$, then

$$
\left.\mathscr{E}_{3}\right|_{C} \cong \mathcal{O} \oplus \mathcal{O}(-1)^{\oplus 2}, \quad V / \mathscr{E}_{3} \cong \mathcal{O}^{\oplus 2} \oplus \mathcal{O}(1)^{\oplus 2} .
$$

Associated to $C$, there are canonical subspaces $V_{1} \subset V_{5} \subset V$, such that $V_{1}$ is the intersection of $\mathscr{E}_{3}(x)$ as $x$ runs through all points on $C$ and that $V_{5}$ is generated by $\mathscr{E}_{3}(x)$ as $x$ runs through all points on $C$. If we vary $C \subset X$, we get a line bundle $\mathscr{E}_{1}$ and a vector bundle $\mathscr{E}_{5}$ of rank 5 on $S^{0}$, where $S^{0}$ is space of smooth conics. Let

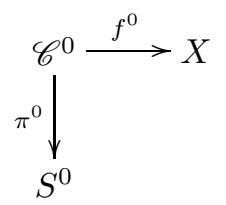

be the universal family. Then we have

$$
\left(\pi^{0}\right)^{*} \mathscr{E}_{1} \subset\left(f^{0}\right)^{*} \mathscr{E}_{3} \subset\left(\pi^{0}\right)^{*} \mathscr{E}_{5} \subset V
$$

By abuse of notation, we omit the "pull-back" and write

$$
\mathscr{E}_{1} \subset \mathscr{E}_{3} \subset \mathscr{E}_{5} \subset V
$$

Hence we have a canonical morphism

$$
\varphi^{0}: S^{0} \rightarrow G(1,5, V),
$$

where $G(1,5, V)$ is the flag variety. We still use $\mathscr{E}_{1} \subset \mathscr{E}_{5} \subset V$ to denote the canonical rank 1 and rank 5 subbundles of $V$ on $G(1,5, V)$. Note that $\eta$ induces

$$
\eta^{\prime}: \mathscr{E}_{1} \otimes\left(\mathscr{E}_{5} / \mathscr{E}_{1}\right) \rightarrow N
$$

Let $S \subset G(1,5, V)$ be the closed subscheme defined by $\eta^{\prime}=0$.

LEMmA A.5. Notations and assumptions as above, the following are true:

(i) $S \supset \operatorname{Im}\left(\varphi^{0}\right)$;

(ii) The morphism $S^{0} \rightarrow S$ induces inclusion $S^{0}(\mathbb{C}) \subset S(\mathbb{C})$.

Proof. Given a smooth conic $C \subset X$, one easily checks that $V_{1} \otimes V_{5} / V_{1} \rightarrow N$ vanishes. This proves (i). To prove (ii), let $V_{1} \subset V_{5} \subset V$ be a pair with $\eta\left(V_{1} \otimes V_{5} / V_{1}\right)=$ 0 .

$$
\begin{aligned}
\left\{x \in X: V_{1} \subset \mathscr{E}_{3}(x) \subset V_{5}\right\} & =\left\{V_{3} / V_{1} \in G\left(2, V_{5} / V_{1}\right): \wedge^{2}\left(V_{3} / V_{1}\right) \rightarrow N \text { is } 0\right\} \\
& =G\left(2, V_{5} / V_{1}\right) \cap \mathbb{P}^{2} \text { in } \mathbb{P}^{5} \\
& =\text { conic on } X
\end{aligned}
$$


The last equality is because otherwise $X$ contains a $\mathbb{P}^{2}$ which is impossible. Hence $C$ is uniquely determined by the pair $V_{1} \subset V_{5}$.

Proposition A.6. The following are true.

(i) The scheme $S$ has pure dimension 2. In particular, $S$ is local complete intersection and hence reduced.

(ii) $S^{0} \rightarrow S$ is open immersion and $S^{0} \subset S$ is dense.

(iii) Over $S$ there is a canonical family $\mathscr{C}$ of conics on $X$ which is constructed in the following way:

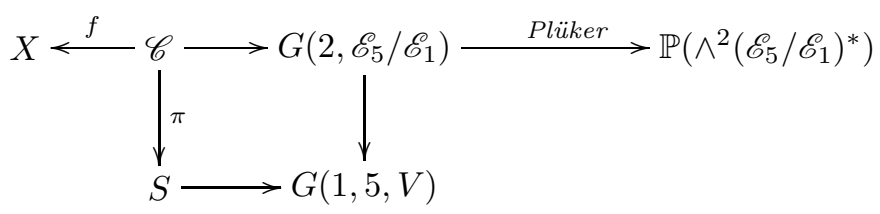

where

$$
\mathscr{C}=G\left(2,\left.\left(\mathscr{E}_{5} / \mathscr{E}_{1}\right)\right|_{S}\right) \cap\{\lambda=0\} \text { in } \mathbb{P}\left(\left.\wedge^{2}\left(\mathscr{E}_{5} / \mathscr{E}_{1}\right)^{*}\right|_{S}\right)
$$

with $\lambda:\left.\left.\mathscr{L}_{\text {taut }}\right|_{S} \rightarrow \wedge^{2}\left(\mathscr{E}_{5} / \mathscr{E}_{1}\right)\right|_{S} \rightarrow N$ being the natural homomorphism. Furthermore, we have $\mathscr{C}^{0}=\left.\mathscr{C}\right|_{S^{0}}$.

Proof. The construction in (iii) is just the proof of the second part of Lemma A.5 in a family. The expected dimension of $S$ is 2 , hence $\operatorname{dim} S \geq 2$. If $S$ is not of pure dimension 2 , there would be a 3 -dimensional family of broken conics on $X$ which is impossible. Hence we proved (i). The fact that the broken conics on $X$ form a 1-dimensional family implies that $S^{0} \rightarrow S$ is open and dense. This proves (ii).

A.7. Consider the natural morphism

$$
\phi: S \hookrightarrow G(1,5, V) \rightarrow G(1, V)=\mathbb{P}\left(V^{*}\right) \cong \mathbb{P}^{6}
$$

where the second morphism is the natural projection.

Proposition A.8. We have the following.

(i) The image of $\phi$ can be characterized in the following way

$$
\begin{aligned}
\operatorname{Im}(\phi) & =\left\{x \in \mathbb{P}\left(V^{*}\right): \operatorname{rank}_{x}\left(\mathscr{E}_{1} \otimes V / \mathscr{E}_{1} \rightarrow N\right) \leq 2\right\} \\
& =\left\{x \in \mathbb{P}\left(V^{*}\right): \operatorname{rank}_{x}\left(\mathscr{E}_{1} \otimes V / \mathscr{E}_{1} \rightarrow N\right)=2\right\}
\end{aligned}
$$

(ii) $\phi$ is a closed immersion.

(iii) On S, we have

$$
\mathscr{E}_{1} \otimes\left(V / \mathscr{E}_{5}\right) \cong \mathscr{N}_{2} \hookrightarrow N
$$

is a rank 2 subbundle of $N$. This gives

$$
\rho: S \rightarrow G(2, N)=\mathbb{P}(N) \cong \mathbb{P}^{2} .
$$

Proof. If there is a 1-dimensional subspace $V_{1} \subset V$ such that $\operatorname{rank}\left(V_{1} \otimes V / V_{1} \rightarrow\right.$ $N)=1$, then there is a 6 -dimensional subspace $V_{6} \subset V$ such that

$$
\eta\left(V_{1} \otimes V_{6} / V_{1}\right)=0
$$


Then $G\left(2, V_{6} / V_{1} ; \eta\right) \subset X$ where

$$
\begin{aligned}
G\left(2, V_{6} / V_{1} ; \eta\right) & =\left\{E / V_{1} \subset V_{6} / V_{1}: \eta\left(\wedge^{2} E\right)=0 \text { and } \operatorname{dim} E=3\right\} \\
& =G(2,5) \cap H_{1} \cap H_{2} \cap H_{3}
\end{aligned}
$$

This implies that $X=G(2,5) \cap H_{1} \cap H_{2} \cap H_{3}$ and hence $X$ is a Fano threefold of index 2 and degree 5. This is a contradiction. Now suppose we are given $V_{1} \subset V$ with $\operatorname{rank}\left(V_{1} \otimes V / V_{1} \rightarrow N\right)=2$. Then there is a unique $V_{5} \subset V$ such that $\eta\left(V_{1} \otimes V_{5} / V_{1}\right)=0$. This proves (i). Let $Z \subset \mathbb{P}\left(V^{*}\right)$ be the closed subscheme defined by the degeneration of the homomorphism $\mathscr{E}_{1} \otimes V / \mathscr{E}_{1} \rightarrow N$. The above argument also shows that $S \rightarrow Z$ is isomorphism hence we have (ii). The rank condition in (i) implies that $\mathscr{N}_{2}=$ $\operatorname{Im}\left(\mathscr{E}_{1} \otimes V / \mathscr{E}_{1} \rightarrow N\right)$ is a rank 2 subbundle of $N$. Hence (iii) follows easily.

A.9. There is a natural linear map

$$
\operatorname{Sym}^{3}\left(\wedge^{2} V^{*}\right) \longrightarrow \wedge^{6} V^{*} \cong V
$$

This induces

$$
V^{*} \stackrel{\tau}{\longrightarrow} \operatorname{Sym}^{3}\left(\wedge^{2} V\right) \stackrel{\operatorname{Sym}^{3}(\eta)}{\longrightarrow} \operatorname{Sym}^{3}(N)
$$

which induces

$$
\phi^{\prime}: \mathbb{P}(N) \cong \mathbb{P}^{2} \longrightarrow \mathbb{P}\left(V^{*}\right)=G(1, V)
$$

Eventually, we want to show that $S \stackrel{\rho}{\longrightarrow} \mathbb{P}(N) \stackrel{\phi^{\prime}}{\longrightarrow} \mathbb{P}\left(V^{*}\right)$ is the same as $\phi$.

A.10. Let $\underline{\alpha}=\left(\alpha_{1}, \alpha_{2}, \ldots, \alpha_{2 n}\right)$ be an ordered set of distinct symbols. A set $\Lambda=$ $\left\{\Lambda_{1}, \Lambda_{2}, \ldots, \Lambda_{n}\right\}$ is called a 2-partition of $\underline{\alpha}$ and we write $\Lambda \prec_{2} \underline{\alpha}$, if $\Lambda_{i}=\left(\lambda_{i, 1}, \lambda_{i, 2}\right)$ with $\lambda_{i, 1}<\lambda_{i, 2}$ and $\bigcup_{i=1}^{n} \Lambda_{i}=\underline{\alpha}$ as sets. Then we define the sign of $\Lambda$ to be

$$
\operatorname{sign}(\Lambda)=\operatorname{sign}_{\underline{\alpha}}(\Lambda)=\operatorname{sign}\left(\begin{array}{cccccc}
\alpha_{1} & \alpha_{2} & \alpha_{3} & \alpha_{4} & \cdots & \alpha_{2 n} \\
\lambda_{1,1} & \lambda_{1,2} & \lambda_{2,1} & \lambda_{2,2} & \cdots & \lambda_{n, 2}
\end{array}\right)
$$

Then the natural linear map $\tau: V^{*} \rightarrow \operatorname{Sym}^{3}\left(\wedge^{2} V\right)$ is given by

$$
\tau\left(e_{i}^{*}\right)=(-1)^{i-1} \sum_{\Lambda \prec_{2}(1,2, \ldots, \hat{i}, \ldots, 7)} \operatorname{sign}(\Lambda) e_{\Lambda_{1}} e_{\Lambda_{2}} e_{\Lambda_{3}}
$$

where $\left\{e_{1}, \ldots, e_{7}\right\}$ is a basis of $V$ and $\left\{e_{1}^{*}, \ldots, e_{7}^{*}\right\}$ is the dual basis of $V^{*} ; e_{\Lambda_{i}}=$ $e_{\lambda_{i, 1}} \wedge e_{\lambda_{i, 2}}$. So the morphism $\phi^{*}: V^{*} \rightarrow \operatorname{Sym}^{3}(N)$ is given by

$$
e_{i}^{*} \mapsto(-1)^{i-1} \sum_{\Lambda \prec_{2}(1,2, \ldots, \hat{i}, \ldots, 7)} \operatorname{sign}(\Lambda) \eta\left(e_{\Lambda_{1}}\right) \eta\left(e_{\Lambda_{2}}\right) \eta\left(e_{\Lambda_{3}}\right)
$$

Lemma A.11. For any linear functional $l: N \rightarrow \mathbb{C}$, i.e. a point $[l] \in \mathbb{P}(N)$, let $v \in V$ be given by

$$
v=\sum_{i=1}^{7}(-1)^{i-1} e_{i} \sum_{\Lambda \prec 2(1,2, \ldots, \hat{i}, \ldots, 7)} \operatorname{sign}(\Lambda) l \circ \eta\left(e_{\Lambda_{1}}\right) l \circ \eta\left(e_{\Lambda_{2}}\right) l \circ \eta\left(e_{\Lambda_{3}}\right)
$$


Then $\phi^{\prime}([l])=[\mathbb{C} v]$ and $l \circ \eta\left(v^{\prime} \wedge v\right)=0$ for all $v^{\prime} \in V$.

Proof. The fact that $\phi^{\prime}([l])=[\mathbb{C} v]$ follows directly from the above explicit computations. To prove the second equation, we only need to do so for $v^{\prime}=e_{j}$. By symmetry, we only need to do the case $j=1$. To make the computation easier to understand, we use the symbol $1^{\prime}$ to replace $j=1$.

$$
\begin{aligned}
& l \circ \eta\left(e_{1} \wedge v\right) \\
& =l \circ \eta\left(e_{1} \wedge v\right) \\
& =\sum_{i=1}^{7}(-1)^{i-1} l \circ \eta\left(e_{1^{\prime}} \wedge e_{i}\right) \sum_{\Lambda \prec 2(1,2, \ldots, \hat{i}, \ldots, 7)} \operatorname{sign}(\Lambda) l \circ \eta\left(e_{\Lambda_{1}}\right) l \circ \eta\left(e_{\Lambda_{2}}\right) l \circ \eta\left(e_{\Lambda_{3}}\right) \\
& =\sum_{i=1}^{7} \sum_{\Lambda \prec 2}(-1)^{i-1} \operatorname{sign}\left(\begin{array}{ccccccc}
1^{\prime} & i & 1 & \cdots & \hat{i} & \ldots & 7 \\
1^{\prime} & i & \lambda_{1,1} & \cdots & . & \ldots & \lambda_{3,2}
\end{array}\right) \cdot l \circ \eta\left(e_{1^{\prime}} \wedge e_{i}\right) \\
& \cdot l \circ \eta\left(e_{\Lambda_{1}}\right) \cdot l \circ \eta\left(e_{\Lambda_{2}}\right) \cdot l \circ \eta\left(e_{\Lambda_{3}}\right) \\
& =\sum_{i=1}^{7} \sum_{\Lambda \prec 2(1,2, \ldots, \hat{i}, \ldots, 7)} \operatorname{sign}\left(\begin{array}{ccccccc}
1^{\prime} & 1 & 2 & \cdots & i & \cdots & 7 \\
1^{\prime} & i & \lambda_{1,1} & & \cdots & & \lambda_{3,2}
\end{array}\right) l \circ \eta\left(e_{1^{\prime}} \wedge e_{i}\right) \\
& \cdot l \circ \eta\left(e_{\Lambda_{1}}\right) \cdot l \circ \eta\left(e_{\Lambda_{2}}\right) \cdot l \circ \eta\left(e_{\Lambda_{3}}\right) \\
& =\sum_{\Lambda^{\prime} \prec_{2}\left(1^{\prime}, 1, \ldots, 7\right)} \operatorname{sign}\left(\Lambda^{\prime}\right) \prod_{i=1}^{4} l \circ \eta\left(e_{\Lambda_{i}^{\prime}}\right) \\
& =-\sum_{\Lambda^{\prime} \prec_{2}\left(1,1^{\prime}, \ldots, 7\right)} \operatorname{sign}\left(\Lambda^{\prime}\right) \prod_{i=1}^{4} l \circ \eta\left(e_{\Lambda_{i}^{\prime}}\right) \\
& =-l \circ \eta\left(e_{1} \wedge v\right)
\end{aligned}
$$

This implies that $l \circ \eta\left(e_{1} \wedge v\right)=0$.

Proposition A.12. The following are true.

(i) The composition $S \stackrel{\rho}{\longrightarrow} \mathbb{P}(N) \stackrel{\phi^{\prime}}{\longrightarrow} \mathbb{P}\left(V^{*}\right)$ is the same as $\phi: S \rightarrow \mathbb{P}\left(V^{*}\right)$.

(ii) The morphism $\rho: S \rightarrow \mathbb{P}(N)$ is isomorphism.

Proof. Let $s=\left[V_{1} \subset V_{5}\right] \in S$ be an arbitrary closed point, then we get $N_{2}=$ $\eta\left(V_{1} \otimes V / V_{1}\right) \subset N$ is a 2-dimensional subspace with $N_{2} \cong V_{1} \otimes V / V_{5}$. Then $\rho(s)=[l]$ where $l: N \rightarrow N / N_{2} \cong \mathbb{C}$. We choose a basis of $V$ such that $V_{1}=\mathbb{C} e_{1}$ and $\left\{e_{1}, \ldots, e_{5}\right\}$ form a basis of $V_{5}$. Then by (15), we get

$$
\begin{aligned}
v & =\sum_{i=1}^{7}(-1)^{i-1} e_{i} \sum_{\substack{\Lambda \prec_{2}(1,2, \ldots, \hat{i}, \ldots, 7)\\
}} \operatorname{sign}(\Lambda) l \circ \eta\left(e_{\Lambda_{1}}\right) l \circ \eta\left(e_{\Lambda_{2}}\right) l \circ \eta\left(e_{\Lambda_{3}}\right) \\
& =e_{1} \sum_{\Lambda \prec_{2}(2,3, \ldots, 7)} \operatorname{sign}(\Lambda) l \circ \eta\left(e_{\Lambda_{1}}\right) l \circ \eta\left(e_{\Lambda_{2}}\right) l \circ \eta\left(e_{\Lambda_{3}}\right)
\end{aligned}
$$

the second equality holds since $l \circ \eta\left(e_{1} \wedge w\right)=0$ for all $w \in V$. It follows that $\phi(s)=\phi^{\prime} \circ \rho(s)$. Since $S$ is reduced, we get $\phi=\phi^{\prime} \circ \rho$ by Hilbert Nullstellensatz. This proves (i). Then $\rho: S \rightarrow \mathbb{P}(N)$ is bijective on points and has smooth image. Then $\rho$ has to be an isomorphism and hence (ii). 
A.13. We have already constructed the canonical family $\mathscr{C}$ of conics on $X$ over the base scheme $S$. Now we want to study more details about the conic bundle $\mathscr{C} \rightarrow S$. On $S$, we have an induced homomorphism $\wedge^{2}\left(\mathscr{E}_{5} / \mathscr{E}_{1}\right) \rightarrow N$. Let $\mathscr{F}$ be its kernel which is a rank 3 vector bundle on $S$. Then we have the following short exact sequence,

$$
0 \longrightarrow \mathscr{F} \longrightarrow \wedge^{2}\left(\mathscr{E}_{5} / \mathscr{E}_{1}\right) \longrightarrow 0
$$

By construction, $\mathscr{C}=G\left(2, \mathscr{E}_{5} / \mathscr{E}_{1}\right) \cap G(1, \mathscr{F})$ in $\mathbb{P}\left(\wedge^{2}\left(\mathscr{E}_{5} / \mathscr{E}_{1}\right)^{*}\right)=G\left(1, \wedge^{2}\left(\mathscr{E}_{5} / \mathscr{E}_{1}\right)\right)$. Then we have the following commutative diagram

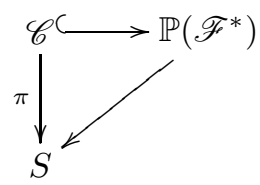

The divisor $G\left(2, \mathscr{E}_{5} / \mathscr{E}_{1}\right)$ on $G\left(1, \wedge^{2}\left(\mathscr{E}_{5} / \mathscr{E}_{1}\right)\right)$ is given be vanishing of the section

$$
\sigma: \operatorname{Sym}^{2}(\mathscr{L}) \longrightarrow \operatorname{Sym}^{2}\left(\wedge^{2}\left(\mathscr{E}_{5} / \mathscr{E}_{1}\right)\right) \longrightarrow \wedge^{4}\left(\mathscr{E}_{5} / \mathscr{E}_{1}\right)
$$

Here $\mathscr{L} \rightarrow \wedge^{2}\left(\mathscr{E}_{5} / \mathscr{E}_{1}\right)$ is the tautological rank 1 subbundle on the scheme $G\left(1, \wedge^{2}\left(\mathscr{E}_{5} / \mathscr{E}_{1}\right)\right)$. Then we have

$$
\begin{aligned}
\left.\sigma\right|_{\mathbb{P}\left(\mathscr{F}^{*}\right)} & \in \mathrm{H}^{0}\left(\mathbb{P}\left(\mathscr{F}^{*}\right), \mathscr{L}^{-2} \otimes \wedge^{4}\left(\mathscr{E}_{5} / \mathscr{E}_{1}\right)\right) \\
& =\mathrm{H}^{0}\left(S, \pi_{*}\left(\mathscr{L}^{-2}\right) \otimes \wedge^{4}\left(\mathscr{E}_{5} / \mathscr{E}_{1}\right)\right) \\
& =\mathrm{H}^{0}\left(S, \operatorname{Sym}^{2}\left(\mathscr{F}^{*}\right) \otimes \wedge^{4}\left(\mathscr{E}_{5} / \mathscr{E}_{1}\right)\right) \\
& \subset \operatorname{Hom}\left(\mathscr{F}, \mathscr{F}^{*} \otimes \wedge^{4}\left(\mathscr{E}_{5} / \mathscr{E}_{1}\right)\right)
\end{aligned}
$$

It is a basic fact, see [29], that the degeneration divisor or discriminant $\Delta \subset S$ is given by the vanishing of

$$
\operatorname{det}\left(\left.\sigma\right|_{\mathbb{P}\left(\mathscr{F}^{*}\right)}\right) \in \mathrm{H}^{0}\left(S, \operatorname{det}\left(\mathscr{F}^{*}\right)^{\otimes 2} \otimes \operatorname{det}\left(\mathscr{E}_{5} / \mathscr{E}_{1}\right)^{\otimes 3}\right)
$$

Namely, $\Delta=\operatorname{div}\left(\operatorname{det}\left(\left.\sigma\right|_{\mathbb{P}\left(\mathscr{F}^{*}\right)}\right)\right)$. This implies that the divisor class of $\Delta$ is $-2 c_{1}(\mathscr{F})+$ $3 c_{1}\left(\mathscr{E}_{5} / \mathscr{E}_{1}\right)$. By $(16)$, we know that $c_{1}(\mathscr{F})=c_{1}\left(\wedge^{2}\left(\mathscr{E}_{5} / \mathscr{E}_{1}\right)\right)=3 c_{1}\left(\mathscr{E}_{5} / \mathscr{E}_{1}\right)$. Hence we eventually have

$$
\Delta \sim-3 c_{1}\left(\mathscr{E}_{5} / \mathscr{E}_{1}\right)
$$

LEMmA A.14. On $S \cong \mathbb{P}(N) \cong \mathbb{P}^{2}$ we have the following relations on divisor classes:

$$
c_{1}\left(\mathscr{E}_{1}\right)=-3 h, \quad c_{1}\left(\mathscr{E}_{5}\right)=-5 h
$$

where $h$ is the class of a line on $\mathbb{P}^{2}$.

Proof. Since $\mathscr{E}_{1} \cong \phi^{*} \mathcal{O}_{\mathbb{P}\left(V^{*}\right)}(-1) \cong \mathcal{O}_{\mathbb{P}^{2}}(-3)$, we get $c_{1}\left(\mathscr{E}_{1}\right)=-3 h$. We also have $\mathscr{E}_{1} \otimes V / \mathscr{E}_{5} \cong \mathscr{N}_{2} \subset N$, this implies that $c_{1}\left(\mathscr{E}_{5}\right)=-5 h$.

Proposition A.15. The degeneration divisor $\Delta$ of the conic bundle $\pi: \mathscr{C} \rightarrow S$ is equivalent to $6 h$, where $h$ is the class of a line on $S \cong \mathbb{P}^{2}$. 
A.16. In this section, we would like to study the ramification of the natural map $f: \mathscr{C} \rightarrow X$. To do this, we consider the following diagram.

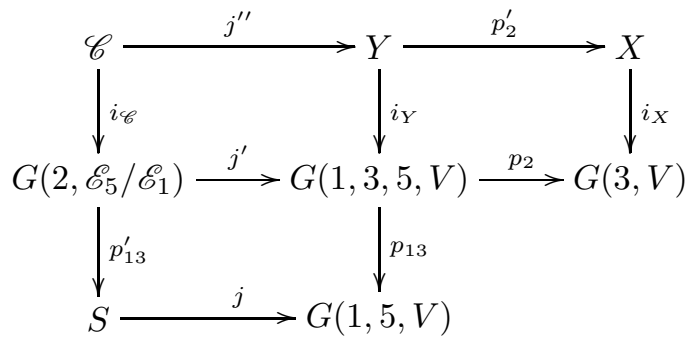

where all squares are fiber-product squares. The closed immersion $i_{X}$ gives the following short exact sequence

$$
\left.\left.0 \longrightarrow \wedge^{2} \mathscr{E}_{3}\right|_{X} \otimes N^{*} \longrightarrow \Omega_{G(3, V)}^{1}\right|_{X} \stackrel{i_{X}^{*}}{\longrightarrow} \Omega_{X}^{1} \longrightarrow 0
$$

The morphism $p_{2}^{\prime}$ realizes $Y$ as a $G(1,3) \times G(2,4)$-bundle over $X$ and hence $Y$ is smooth. We have a similar sequence for $i_{Y}$.

$$
\left.0 \longrightarrow \wedge^{2} \mathscr{E}_{3}\right|_{Y} \otimes N^{*} \longrightarrow \Omega_{G(1,3,5, V)}^{1} \stackrel{i_{Y}^{*}}{\longrightarrow} \Omega_{Y}^{1} \longrightarrow 0
$$

Note that $j^{\prime \prime}: \mathscr{C} \rightarrow Y$ is given by the vanishing of $\mathscr{E}_{1} \otimes\left(\mathscr{E}_{5} / \mathscr{E}_{3}\right) \rightarrow N$. This gives

$$
\left.\left.0 \longrightarrow \mathscr{E}_{1} \otimes\left(\mathscr{E}_{5} / \mathscr{E}_{3}\right)\right|_{\mathscr{C}} \otimes N^{*} \longrightarrow \Omega_{Y}^{1}\right|_{\mathscr{C}} \stackrel{j^{\prime \prime *}}{\longrightarrow} \Omega_{\mathscr{C}}^{1} \longrightarrow 0
$$

We put all the above sequences together and get the following commutative diagram.

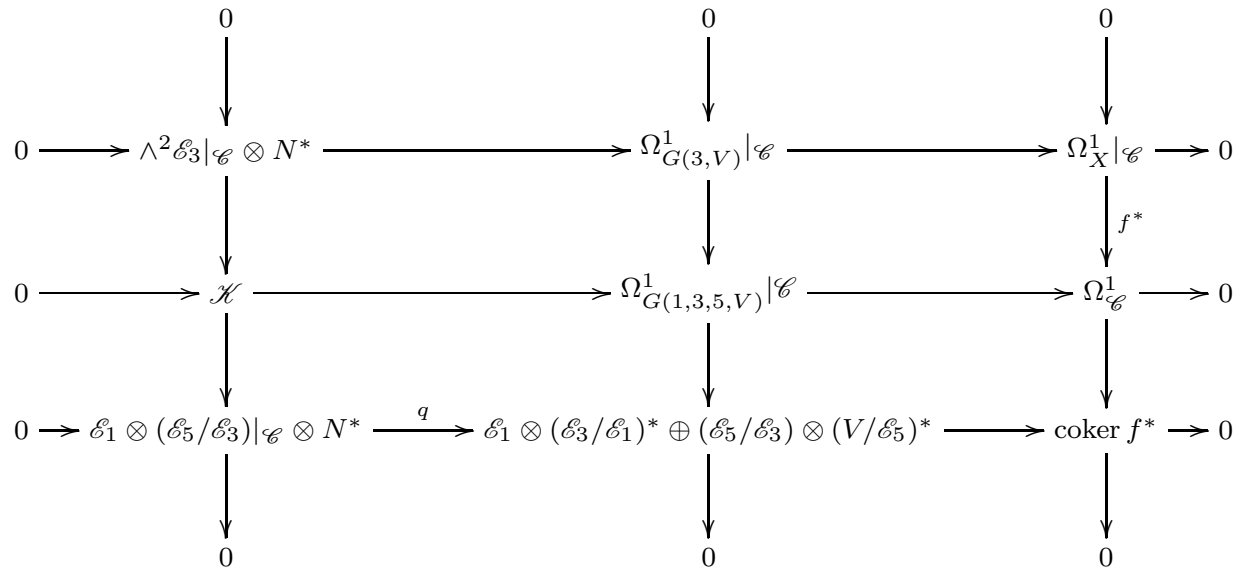

It follows that the ramification divisor $R$ of the morphism of $f: \mathscr{C} \rightarrow X$ is given by $R=\operatorname{div}(\operatorname{det}(q))$, where

$\operatorname{det}(q) \in \mathrm{H}^{0}\left(\mathscr{C}, \operatorname{det}\left(\left.\left(\mathscr{E}_{1} \otimes \mathscr{E}_{5} / \mathscr{E}_{3}\right)^{*}\right|_{\mathscr{C}} \otimes N\right) \otimes \operatorname{det}\left(\mathscr{E}_{1} \otimes\left(\mathscr{E}_{3} / \mathscr{E}_{1}\right)^{*} \oplus\left(\mathscr{E}_{5} / \mathscr{E}_{3}\right) \otimes\left(V / \mathscr{E}_{5}\right)^{*}\right)\right)$ It follows easily that $R \sim \pi^{*}\left(-3 c_{1}\left(\mathscr{E}_{1}\right)+c_{1}\left(\mathscr{E}_{5}\right)\right)$.

Proposition A.17. The ramification divisor $R$ of the morphism $f: \mathscr{C} \rightarrow X$ can be written as $R=\pi^{*} \operatorname{div}\left(s_{0}\right)$ as divisors. Here we identify $S$ with $\mathbb{P}^{2}$ and $s_{0} \in$ $\mathrm{H}^{0}\left(\mathbb{P}^{2}, \mathcal{O}_{\mathbb{P}^{2}}(4)\right)$. 


\section{REFERENCES}

[1] A. Beauville, Variétés de Prym et jacobiennes intermédiaires, Annales Scientifiques de l'É.N.S. $4^{\text {e }}$ série, 10:3 (1977), p. 309-391.

[2] M. Beltrametti, On the Chow group and the intermediate Jacobian of a conic bundle, Ann. Math. Pura Appl. (4), 141 (1985), p. 331-351.

[3] I. Biswas and D. S. NagaraJ, Vector bundles over a nondegenerate conic, J. Aust. Math. Soc., 86 (2009), pp. 145-154.

[4] F. Campana, Connexité rationnelle des variétés de Fano, Ann. Sci. E.N.S., 25 (1992), pp. 539 545.

[5] C. H. Clemens and P. A. Griffiths, The intermediate Jacobian of cubic threefold, Annals of Math., 95:2 (1972), pp. 281-356.

[6] I. Coskun And J. StaRR, Rational curves on smooth cubic hypersurfaces, Int. Math. Res. Not., 24 (2009), pp. 4626-4641.

[7] S. D. CutKosky, On Fano 3-folds, Mnuscripta Math., 64 (1989), pp. 189-204.

[8] D. Eisenbud And J. Harris, On varieties of minimal degree (a centennial account), Proceedings of Symposia in Pure Mathematics, volume 46.

[9] T. Ekedahl, N. I. Shepherd-Barron and R. L. Taylor, A conjecture on the existence of compact leaves of algebraic foliations, Preprint, April 1999.

[10] S. Yu.ĖndRYushKa, The intermediate Jacobian of a three-dimensional conic bundle is a Prym variety, Math. USSR. Sbornik, 50:1, 1985.

[11] M. Furushima and N. Nakayama, The family of lines on the Fano threefold $V_{5}$, Nagoya Math. J., 116 (1989), pp. 111-122.

[12] T. Graber, J. HARRis, ANd J. Starr, Families of rationally connected varieties, J. Amer. Math. Soc., 16:1 (2003), pp. 57-67.

[13] P. Griffiths and J. Harris, Principles of algebraic geometry, John Wiley \& Sons, Inc. 1978.

[14] R. Hartshorne, Curves with high self-intersection on algebraic surfaces, Publ. Math. de l'I.H.É.S., 36 (1969), pp. 111-125.

[15] J. Harris, M. Roth and J. StarR, Rational curves on hypersurfaces of low degree, J. Reine Angew. Math., 571 (2004), pp. 73-106.

[16] D. HuYBrechts And M. LehN, The geometry of moduli spaces of sheaves, Aspects of Mathematics, 31 Friedr. Vieweg Sohn, Braunschweig, 1997.

[17] A. Iliev and D. Markushevich, Parametrization of Sing $\Theta$ for a Fano threefold of genus 7 by moduli of vector bundles, Arxiv:math/0403122v3.

[18] V. A. Iskovskikh, Fano 3-folds. II, Math. USSR Izvestiya, 12:3 (1978).

[19] V. A. Iskovskikn, Double projection from a line on Fano threefolds of the first kind, Math. USSR Sbornik, 66:1 (1990).

[20] V. A. Iskovskikh and Yu. G. Prokhorov, Fano Varieties, Algebraic Geometry V, EMS 47, Springer, Berlin, 1999.

[21] V. KANEV, Intermediate Jacobians and Chow groups of threefolds with a pencil of Del Pezzo surfaces, Annali di Matematica Pura ed Applicata (IV), Vol. CLIV (1989), pp. 13-48.

[22] B. Kim And R. PANDhARIPANDE, The connectedness of the moduli space of maps to homogeneous spaces, in Symplectic geometry and mirror symmetry, pp. 187-201, World Sci. Publ., 2001.

[23] J. Kollár, Rational curves on algebraic varieties, Ergeb. der Math. Grenzgeb., vol. 32, PringerVerlag, Berlin, 1996.

[24] J. KolláR, Y. MiYAOKA And S. MoRI, Rational connectedness and boundedness of Fano manifolds, J. Diff. Geom., 1 (1992), pp. 765-769.

[25] J. Kollár and F.-O. Schreyer, Real Fano 3-folds of type $V_{22}$, In The Fano Conference, pp. 515-531, Turin, 2004, University Torino.

[26] J. Li AND G. TiAn, Virtual moduli cycles and Gromov-Witten invariants of algebraic varieties, J. Amer. Math. Soc., 11 (1998), pp. 119-174.

[27] D. Logachev, Fano threefolds of genus 6, November, 1982. Arxiv:math/0407147v1.

[28] S. Mukai, Fano threefolds, in Complex Projective Geometry, London Math. Soc. Lecture Notes, Ser. 179, Cambridge University Press, 1992, pp. 255-263.

[29] V. G. Sarkisov, On conic bundle structures, Math. USSR Izvestiya, 20:2 (1982).

[30] A. Sснмiтt, On the topology of flops of (-2)-curves, Indag. Mathem., N.S., 9:3 (1998), pp. 443450.

[31] E. SERnESI, Deformations of algebraic schemes, Grundlehren der mathematischen Wissenschaften, vol. 334, Springer-Verlag, 2006.

[32] M. Shen, Foliations and Rational Connectedness in Positive Characteristic, J. Alg. Geom., 19 (2010), pp. 531-553. 
[33] J. StarR, Rational curves on hypersurfaces, PhD Thesis, Harvard University, 2000.

[34] J. Starr, Brauer groups and Galois cohomology of function fields of varieties, Preprint 2009.

[35] J. F. Thomsen, Irriducibility of $\bar{M}_{0, n}(g / p, \beta)$, Internat. J. Math., 9 (1998), pp. 367-376.

[36] A. N. Tyurin, Five lectures on three-dimensional varieties, Russ. Math. Surveys, 27 (1972), pp. $1-53$.

[37] G. E. Welters, Abel-Jacobi isogenies for certain types of Fano threefolds, Mathematical Centre Tracts 141, Amsterdam 1981.

[38] O. Zariski And P. SAmuel, Commutative algebra, Volume II, GTM 29, Springer-Verlag. 